JOURNAL OF THE

AMERICAN MATHEMATICAL SOCIETY

Volume 19, Number 3, Pages 551-578

S 0894-0347(05)00516-3

Article electronically published on December 22, 2005

\title{
NON-COMPUTABLE JULIA SETS
}

\author{
M. BRAVERMAN AND M. YAMPOLSKY
}

\section{Summary of the Paper}

Polynomial Julia sets have emerged as the most studied examples of fractal sets generated by a dynamical system. Apart from the beautiful mathematics, one of the reasons for their popularity is the beauty of the computer-generated images of such sets. The algorithms used to draw these pictures vary; the most naïve work by iterating the center of a pixel to determine if it lies in the Julia set. Milnor's distance-estimator algorithm [Mil] uses classical complex analysis to give a one-pixel estimate of the Julia set. This algorithm and its modifications work quite well for many examples, but it is well known that in some particular cases computation time will grow very rapidly with increase of the resolution. Moreover, there are examples, even in the family of quadratic polynomials, when no satisfactory pictures of the Julia set exist. In this paper we study computability properties of Julia sets of quadratic polynomials. Under the definition we use, a set is computable, if, roughly speaking, its image can be generated by a computer with an arbitrary precision. Under this notion of computability we show:

Main Theorem. There exists a parameter value $c \in \mathbb{C}$ such that the Julia set of the quadratic polynomial $f_{c}(z)=z^{2}+c$ is not computable.

The structure of the paper is as follows. In the Introduction we discuss the question of computability of real sets and make the relevant definitions. Further in this section we briefly introduce the reader to the main concepts of Complex Dynamics and discuss the properties of Julia sets relevant to us. In the end of the Introduction, we outline the conceptual idea of the proof of the Main Theorem. Section 3 contains the technical lemmas on which the argument is based. In $\S 4$ we complete the proof.

\section{INTRODUCTION}

\subsection{Introduction to the computability of real sets.}

Classical computability. The computability theory in general allows us to classify problems into the tractable ("computable") and intractable ("non-computable") All the common computational tasks such as integer operations, list sorting, etc. are easily seen to be computable. On the other hand, there are many non-computable problems.

Received by the editors June 28, 2004.

2000 Mathematics Subject Classification. Primary 37F50; Secondary 68Q17.

The first author's research is supported by an NSERC CGS scholarship.

The second author's research is supported by an NSERC operating grant.

(C)2005 American Mathematical Society Reverts to public domain 28 years from publication 
In the formal setting for the study of computability theory computations are performed by objects called Turing Machines. Turing Machines were introduced in 1936 by Alan Turing (see [Tur]) and are accepted by the scientific community as the standard model of computation. The Turing Machine (TM in short) is capable of solving exactly the same problems as an ordinary computer. Most of the time, one can think of the TM as a computer program written in any programming language. It is important to mention that there are only countably many TMs, which can be enumerated in a natural way. See Sip for a formal discussion on TMs. We define computability as follows.

Definition 2.1. We say that a function $f:\{0,1\}^{*} \rightarrow\{0,1\}^{*}$ is computable if there is a TM, which on input string $s$ outputs the string $f(s)$.

We say that the set $L \subset\{0,1\}^{*}$ is computable or decidable if its characteristic function $\chi_{L}:\{0,1\}^{*} \rightarrow\{0,1\}$ is computable.

While most "common" functions are computable, there are uncountably many non-computable functions and undecidable sets. The best-known intractable problems are the Halting Problem and the solvability of a Diophantine equation (Hilbert's 10-th problem); see [Sip and Mat] for more information.

Computability of real functions and sets. In the present paper we are interested in the computability of functions $f: \mathbb{R}^{n} \rightarrow \mathbb{R}$ and subsets of $\mathbb{R}^{n}$, particularly subsets of $\mathbb{R}^{2} \cong \mathbb{C}$. We cannot directly apply Definition 2.1 here, since a real number cannot be represented in general by finite sequences of bits.

Denote by $\mathbb{D}$ the set of the dyadic rationals, that is, rationals of the form $\frac{p}{2^{m}}$. We say that $\phi: \mathbb{N} \rightarrow \mathbb{D}$ is an oracle for a real number $x$, if $|x-\phi(n)|<2^{-n}$ for all $n \in \mathbb{N}$. In other words, $\phi$ provides a good dyadic approximation for $x$. We say that a TM $M^{\phi}$ is an oracle machine if at any step of the computation $M$ is allowed to query the value $\phi(n)$ for any $n$. This definition allows us to define the computability of real functions on compact sets.

Definition 2.2. We say that a function $f:[a, b] \rightarrow[c, d]$ is computable if there exists an oracle TM $M^{\phi}(m)$ such that if $\phi$ is an oracle for $x \in[a, b]$, then on input $m, M^{\phi}$ outputs a $y \in \mathbb{D}$ such that $|y-f(x)|<2^{-m}$.

This definition was first introduced by Grzegorczyk Grz and Lacombe Lac and follows in the tradition of Computable Analysis originated by Banach and Mazur in 1936-1939 (see Maz).

To understand the definition better, the reader without a Computer Science background should think of a computer program with an instruction

$$
\text { READ real number } x \text { WITH PRECISION } n(m) \text {. }
$$

On the execution of this command, a dyadic rational $d$ is input from the keyboard. This number must not differ from $x$ by more than $2^{-n(m)}$ (but otherwise can be arbitrary). The algorithm then outputs $f(x)$ to precision $2^{-n}$.

In other words, with an access to arbitrarily good approximations for $x, M$ should be able to produce an arbitrarily good approximation for $f(x)$. This definition trivially generalizes to domains of higher dimension. See Ko1 for more details. One of the most important properties of computable functions is that

Proposition 2.1. Computable functions are continuous. 
Let $K \subset \mathbb{R}^{k}$ be a compact set. We would like to give a definition for $K$ being computable. Note that saying, by a naïve analogy with Definition 2.1, that $K$ is computable if and only if the characteristic function $\chi_{K}$ is computable does not work here, since by the above proposition only continuous functions can be computable.

We say that a TM $M$ computes the set $K$ if it approximates $K$ in the Hausdorff metric. Recall that the Hausdorff metric is a metric on compact subsets of $\mathbb{R}^{n}$ defined by

$$
d_{H}(X, Y)=\inf \left\{\epsilon>0 \mid X \subset U_{\epsilon}(Y) \text { and } Y \subset U_{\epsilon}(X)\right\},
$$

where $U_{\epsilon}(S)$ is defined as the union of the set of $\epsilon$-balls with centers in $S$.

We introduce a class $\mathcal{C}$ of sets which is dense in the metric $d_{H}$ among the compact sets and which has a natural correspondence to binary strings. Namely $\mathcal{C}$ is the set of finite unions of dyadic balls:

$$
\mathcal{C}=\left\{\bigcup_{i=1}^{n} \overline{B\left(d_{i}, r_{i}\right)} \mid \text { where } d_{i}, r_{i} \in \mathbb{D}\right\} .
$$

The following definition is equivalent to the set computability definition given in Wei] (see also [RW]).

Definition 2.3. We say that a compact set $K \subset \mathbb{R}^{k}$ is computable if there exists a TM $M(m)$, such that on an input $m \in \mathbb{N}$, the machine $M(m)$ outputs an encoding of $C_{m} \in \mathcal{C}$ such that $d_{H}\left(K, C_{m}\right)<2^{-m}$.

To illustrate the robustness of this definition we present the following two equivalent characterizations of computable sets. The first one relates the definition to computer graphics. It is not stated precisely here, but it can be easily made precise. The second one relates the computability of sets to the computability of functions as per Definition 2.2 .

Theorem 2.2. For a compact $K \subset \mathbb{R}^{k}$ the following are equivalent:

(1) $K$ is computable as per Definition 2.3:

(2) (in the case $k=1,2) K$ can be drawn on a computer screen with arbitrarily good precision;

(3) the distance function $d_{K}(x)=\inf \{|x-y| \mid y \in K\}$ is computable as per Definition 2.2 .

In the present paper we are interested in questions concerning the computability of the Julia set $J_{c}=J\left(f_{c}\right)=J\left(z^{2}+c\right)$ (see the next section for the definition). Since there are uncountably many possible parameter values for $c$, and only countably many TMs, we cannot expect for each $c$ to have a machine $M$ such that $M$ computes $J_{c}$. On the other hand, it is reasonable to want $M$ to compute $J_{c}$ with an oracle access to $c$. Define the function $J: \mathbb{C} \rightarrow K^{*}\left(K^{*}\right.$ is the set of all compact subsets of $\mathbb{C}$ ) by $J(c)=J\left(f_{c}\right)$. In a complete analogy to Definition 2.2 we can define

Definition 2.4. We say that a function $f: S \rightarrow K^{*}$ for some bounded set $S \subset \mathbb{R}^{k}$ is computable, if there exits an oracle $\mathrm{TM} M^{\phi}(m)$ such that if $\phi$ is an oracle for $x \in S$, then on input $m, M^{\phi}$ outputs a set $C_{m} \in \mathcal{C}$ such that $d_{H}\left(C_{m}, f(x)\right)<2^{-m}$.

In the case of Julia sets:

Definition 2.5. We say that $J_{c}$ is computable if the function $J: d \mapsto J_{d}$ is computable on the set $\{c\}$. 
The following has been shown (see [Brv1, Ret]):

Theorem 2.3. Denote by $\mathcal{H}$ the set of parameters $c$ for which $J_{c}$ is hyperbolic. Then

(i) $J_{c}$ is computable for all $c \in \mathcal{H}$; moreover,

(ii) the function $J$ is computable on each bounded subset of $\mathcal{H}$.

Our goal in this paper is to show that there are values of $c$ for which $J_{c}$ is not computable under Definition 2.5 which is the weakest possible definition in this setting. We will be using the following version of Theorem 2.1 for set functions.

Theorem 2.4. Suppose that a TM $M^{\phi}$ computes the function $J$ on a set $S$. Then $J$ is continuous on $S$ in the Hausdorff sense.

Proof. Let $c$ be any point in $S$, and let $\varepsilon=2^{-k}$ be given. Let $\phi$ be an oracle for $c$ such that $|\phi(n)-c|<2^{-(n+1)}$ for all $k$. We run $M^{\phi}(k+1)$ with this oracle $\phi$. By the definition of $J$, it outputs a set $L$ which is a $2^{-(k+1)}$ approximation of $J_{c}$ in the Hausdorff metric.

The computation is performed in a finite amount of time. Hence there is an $m$ such that $\phi$ is only queried with parameters not exceeding $m$. Then for any $x$ such that $|x-c|<2^{-(m+1)}, \phi$ is a valid oracle for $x$ up to a parameter value of $m$. In particular, we can create an oracle $\psi$ for $x$ that agrees with $\phi$ on $1,2, \ldots, m$. If $x \in S$, then the execution of $M^{\psi}(k+1)$ will be identical to the execution of $M^{\phi}(k+1)$, and it will output $L$ which has to be an approximation of $J_{x}$. Thus we have

$$
d_{H}\left(J_{c}, J_{x}\right) \leq d_{H}\left(J_{c}, L\right)+d_{H}\left(J_{x}, L\right)<2^{-(k+1)}+2^{-(k+1)}=2^{-k} .
$$

This is true for any $x \in B\left(c, 2^{-(m+1)}\right) \cap S$. Hence $J$ is continuous on $S$.

In the next section we proceed to define Julia sets of rational maps and review their basic properties. In particular, towards the end of the introduction, we will see a mechanism by which the continuity required by Theorem 2.4 may fail.

It should be noted that the question of computability of dynamically generated fractal sets, such as Julia sets, has been discussed by Blum, Cucker, Shub, and Smale in [BCSS]. The definition of set computability used in [BCSS] is, however, quite different from Definition 2.3. The BCSS model allows infinite-precision arithmetic, but requires completely accurate pictures to be generated. Under this definition all Julia sets but the most trivial ones can be shown to be non-computable.

2.2. Julia sets of polynomial mappings. We recall the main definitions of complex dynamics relevant to our result only briefly; a good general reference is the book of Milnor Mil]. For a rational mapping $R$ of degree $\operatorname{deg} R=d \geq 2$ considered as a dynamical system on the Riemann sphere

$$
R: \hat{\mathbb{C}} \rightarrow \hat{\mathbb{C}}
$$

the Julia set is defined as the complement of the set where the dynamics is Lyapunovstable:

Definition 2.6. Denote by $F(R)$ the set of points $z \in \hat{\mathbb{C}}$ having an open neighborhood $U(z)$ on which the family of iterates $\left.R^{n}\right|_{U(z)}$ is equicontinuous. The set $F(R)$ is called the Fatou set of $R$ and its complement $J(R)=\hat{\mathbb{C}} \backslash F(R)$ is the Julia set. 
In the case when the rational mapping is a polynomial

$$
P(z)=a_{0}+a_{1} z+\cdots+a_{d} z^{d}: \mathbb{C} \rightarrow \mathbb{C}
$$

an equivalent way of defining the Julia set is as follows. Obviously, there exists a neighborhood of $\infty$ on $\hat{\mathbb{C}}$ on which the iterates of $P$ uniformly converge to $\infty$. Denoting by $A(\infty)$ the maximal such domain of attraction of $\infty$ we have $A(\infty) \subset$ $F(R)$. We then have

$$
J(P)=\partial A(\infty) .
$$

The bounded set $\hat{\mathbb{C}} \backslash \mathrm{cl} A(\infty)$ is called the filled Julia set and is denoted by $K(P)$; it consists of points whose orbits under $P$ remain bounded:

$$
K(P)=\left\{z \in \hat{\mathbb{C}}\left|\sup _{n}\right| P^{n}(z) \mid<\infty\right\} .
$$

For future reference, let us list in a proposition below the main properties of Julia sets:

Proposition 2.5. Let $R: \hat{\mathbb{C}} \rightarrow \hat{\mathbb{C}}$ be a rational function. Then the following properties hold:

- $J(R)$ is a non-empty compact subset of $\hat{\mathbb{C}}$ which is completely invariant: $R^{-1}(J(R))=J(R)$

- $J(R)=J\left(R^{n}\right)$ for all $n \in \mathbb{N}$;

- $J(R)$ is perfect;

- if $J(R)$ has non-empty interior, then it is the whole of $\hat{\mathbb{C}}$;

- let $U \subset \hat{\mathbb{C}}$ be any open set with $U \cap J(R) \neq \emptyset$. Then there exists $n \in \mathbb{N}$ such that $R^{n}(U) \supset J(R)$;

- periodic orbits of $R$ are dense in $J(R)$.

Let us further comment on the last property. For a periodic point $z_{0}=R^{p}\left(z_{0}\right)$ of period $p$ its multiplier is the quantity $\lambda=\lambda\left(z_{0}\right)=D R^{p}\left(z_{0}\right)$. We may speak of the multiplier of a periodic cycle, as it is the same for all points in the cycle by the Chain Rule. In the case when $|\lambda| \neq 1$, the dynamics in a sufficiently small neighborhood of the cycle is governed by the Mean Value Theorem: when $|\lambda|<1$, the cycle is attracting (super-attracting if $\lambda=0$ ), if $|\lambda|>1$ it is repelling. Both in the attracting and repelling cases, the dynamics can be locally linearized:

$$
\psi\left(R^{p}(z)\right)=\lambda \cdot \psi(z)
$$

where $\psi$ is a conformal mapping of a small neighborhood of $z_{0}$ to a disk around 0. By a classical result of Fatou, a rational mapping has at most finitely many non-repelling periodic orbits. Therefore, we may refine the last statement of Proposition 2.5

- repelling periodic orbits are dense in $J(R)$.

In the case when $|\lambda|=1$, so that $\lambda=e^{2 \pi i \theta}, \theta \in \mathbb{R}$, the simplest to study is the parabolic case when $\theta=n / m \in \mathbb{Q}$, so $\lambda$ is a root of unity. In this case $R^{p}$ is not locally linearizable; it is not hard to see that $z_{0} \in J(R)$. In the complementary situation, two non-vacuous possibilities are considered: the Cremer case, when $R^{p}$ is not linearizable, and the Siegel case, when it is. In the latter case, the linearizing map $\psi$ from (2.2) conjugates the dynamics of $R^{p}$ on a neighborhood $U\left(z_{0}\right)$ to the irrational rotation by angle $\theta$ (the rotation angle) on a disk around the origin. The maximal such neighborhood of $z_{0}$ is called a Siegel disk. Siegel disks will prove crucial to our study and will be discussed in more detail in the next section. 
To conclude the discussion of the basic properties of Julia sets, let us consider the simplest examples of non-linear rational endomorphisms of the Riemann sphere: the quadratic polynomials. Every affine conjugacy class of quadratic polynomials has a unique representative of the form $f_{c}(z)=z^{2}+c$, and the family

$$
f_{c}(z)=z^{2}+c, c \in \mathbb{C}
$$

is often referred to as the quadratic family. For a quadratic map the structure of the Julia set is governed by the behavior of the orbit of the only finite critical point 0 . In particular, the following dichotomy holds:

Proposition 2.6. Let $K=K\left(f_{c}\right)$ denote the filled Julia set of $f_{c}$, and $J=J\left(f_{c}\right)=$ $\partial K$. Then:

- $0 \in K$ implies that $K$ is a connected, compact subset of the plane with connected complement;

- $0 \notin K$ implies that $K=J$ is a planar Cantor set.

The Mandelbrot set $\mathcal{M} \subset \mathbb{C}$ is defined as the set of parameter values $c$ for which $J\left(f_{c}\right)$ is connected.

Continuity of the dependence $c \mapsto J\left(f_{c}\right)$. A natural question to pose for polynomials in the quadratic family is whether the Julia set varies continuously with the parameter $c$. To make sense of this question, recall the definition of the Hausdorff distance $\operatorname{dist}_{H}$ between compact sets $X, Y$ in the plane (2.1). It turns out that the dependence $c \mapsto J\left(f_{c}\right)$ is discontinuous in the Hausdorff distance. For an excellent survey of this problem see the paper of Douady [Do]. The discontinuity which has found the most interesting dynamical applications occurs at parameter values for which $f_{c}$ has a parabolic point. We, however, will employ a more obvious discontinuity which is related to Siegel disks. Let us first note that by a result of Douady and Hubbard [DH1] a quadratic polynomial has at most one non-repelling cycle in $\mathbb{C}$. In particular, there is at most one cycle of Siegel disks.

Proposition 2.7. Let $c_{*} \in \mathcal{M}$ be a parameter value for which $f_{c}$ has a Siegel disk. Then the map $c \mapsto J\left(f_{c}\right)$ is discontinuous at $c_{*}$.

Proof. Let $z_{0}$ be a Siegel periodic point of $f_{c}$ and denote by $\Delta$ the Siegel disk around $\zeta_{0}, p$ its period, and $\theta$ the rotation angle. By the Implicit Function Theorem, there exists a holomorphic mapping $\zeta: U\left(c_{*}\right) \rightarrow \mathbb{C}$ such that $\zeta\left(c_{*}\right)=z_{0}$ and $\zeta(c)$ is fixed under $\left(f_{c}\right)^{p}$. The mapping

$$
\nu: c \mapsto D\left(f_{c}\right)^{p}(\zeta(c))
$$

is holomorphic; hence it is either constant or open. If it is constant, all quadratic polynomials have a Siegel disk. This is not possible: for instance, $f_{1 / 4}$ has a parabolic fixed point and thus no other non-repelling cycles. Therefore, $\nu$ is open, and in particular, there is a sequence of parameters $c_{n} \rightarrow c_{*}$ such that $\zeta\left(c_{n}\right)$ has multiplier $e^{2 \pi i p_{n} / q_{n}}$. Since $\zeta\left(c_{n}\right)$ is parabolic, it lies in the Julia set of $f_{c_{n}}$. Hence

$$
\operatorname{dist}_{H}\left(J\left(f_{c_{*}}\right), J\left(f_{c_{n}}\right)\right)>\operatorname{dist}\left(c_{*}, \partial \Delta\right) / 2
$$

for $n$ large enough.

Thus an arbitrarily small change of the multiplier of the Siegel point may lead to an implosion of the Siegel disk - its inner radius collapses to zero. We make a 
note of an immediate consequence of the above proposition and Theorem 2.4

Proposition 2.8. For any $T M M^{\phi}(n)$ with an oracle for $c \in \mathbb{C}$ denote by $S_{M}$ the set of all values of $c$ for which $M^{\phi}$ computes $J_{c}$. Then $S_{M} \neq \mathbb{C}$.

In other words, a single algorithm for computing all quadratic Julia sets does not exist.

Siegel disks of quadratic maps. Let us discuss in more detail the occurrence of Siegel disks in the quadratic family. For a number $\theta \in[0,1)$ denote by $\left[r_{1}, r_{2}, \ldots\right.$, $\left.r_{n}, \ldots\right], r_{i} \in \mathbb{N} \cup\{\infty\}$ its possibly finite continued fraction expansion:

$$
\left[r_{1}, r_{2}, \ldots, r_{n}, \ldots\right] \equiv \frac{1}{r_{1}+\frac{1}{r_{2}+\frac{1}{\cdots+\frac{1}{r_{n}+\cdots}}}}
$$

Such an expansion is defined uniquely if and only if $\theta \notin \mathbb{Q}$. In this case, the rational convergents $p_{n} / q_{n}=\left[r_{1}, \ldots, r_{n}\right]$ are the closest rational approximants of $\theta$ among the numbers with denominators not exceeding $q_{n}$. In fact, setting $\lambda=e^{2 \pi i \theta}$, we have

$$
\left|\lambda^{h}-1\right|>\left|\lambda^{q_{n}}-1\right| \text { for all } 0<h<q_{n+1}, h \neq q_{n} .
$$

The difference $\left|\lambda^{q_{n}}-1\right|$ lies between $2 / q_{n+1}$ and $2 \pi / q_{n+1}$; therefore the rate of growth of the denominators $q_{n}$ describes how well $\theta$ may be approximated with rationals.

Definition 2.7. The diophantine numbers of order $k$, denoted by $\mathcal{D}(k)$, form the following class of irrationals "badly" approximated by rationals. By definition, $\theta \in \mathcal{D}(k)$ if there exists $c>0$ such that

$$
q_{n+1}<c q_{n}^{k-1} \text {. }
$$

The numbers $q_{n}$ can be calculated from the recurrence relation

$$
q_{n+1}=r_{n+1} q_{n}+q_{n-1}, \text { with } q_{0}=0, q_{1}=1 .
$$

Therefore, $\theta \in \mathcal{D}(2)$ if and only if the sequence $\left\{r_{i}\right\}$ is bounded. Dynamicists call such numbers bounded type (number-theorists prefer constant type). An extreme example of a number of bounded type is the golden mean

$$
\theta_{*}=\frac{\sqrt{5}-1}{2}=[1,1,1, \ldots] .
$$

The set

$$
\mathcal{D}(2+) \equiv \bigcap_{k>2} \mathcal{D}_{k}
$$

has full measure in the interval [0,1). In 1942 Siegel showed:

Theorem 2.9 ([Sie]). Let $R$ be an analytic map with a periodic point $z_{0} \in \hat{\mathbb{C}}$ of period $p$. Suppose the multiplier of the cycle is

$$
\lambda=e^{2 \pi i \theta} \text { with } \theta \in \mathcal{D}(2+) .
$$

Then the local linearization equation (2.2) holds. 
The strongest known generalization of this result was proved by Brjuno in 1972:

Theorem $2.10([\mathrm{Bru}])$. Suppose

$$
B(\theta)=\sum_{n} \frac{\log \left(q_{n+1}\right)}{q_{n}}<\infty .
$$

Then the conclusion of Siegel's Theorem holds.

Note that a quadratic polynomial with a fixed Siegel disk with rotation angle $\theta$ after an affine change of coordinates can be written as

$$
P_{\theta}(z)=z^{2}+e^{2 \pi i \theta} z .
$$

In 1987 Yoccoz Yoc proved the following converse to Brjuno's Theorem:

Theorem 2.11 ( $\overline{\mathrm{Yoc}}]$ ). Suppose that for $\theta \in[0,1)$ the polynomial $P_{\theta}$ has a Siegel point at the origin. Then $B(\theta)<\infty$.

The numbers satisfying (2.4) are called Brjuno numbers; the set of all Brjuno numbers will be denoted $\mathcal{B}$. It is evident that $\cup \mathcal{D}(k) \subset \mathcal{B}$. The sum of the series (2.4) is called the Brjuno function. For us a different characterization of $\mathcal{B}$ will be more useful. Inductively define $\theta_{1}=\theta$ and $\theta_{n+1}=\left\{1 / \theta_{n}\right\}$. In this way,

$$
\theta_{n}=\left[r_{n}, r_{n+1}, r_{n+2}, \ldots\right] \text {. }
$$

We define Yoccoz's Brjuno function as

$$
\Phi(\theta)=\sum_{n=1}^{\infty} \theta_{1} \theta_{2} \cdots \theta_{n-1} \log \frac{1}{\theta_{n}} .
$$

One can verify that

$$
B(\theta)<\infty \Leftrightarrow \Phi(\theta)<\infty .
$$

The value of the function $\Phi$ is related to the size of the Siegel disk in the following way.

Definition 2.8. Let $P(\theta)$ be a quadratic polynomial with a Siegel disk $\Delta_{\theta} \ni 0$. Consider a conformal isomorphism $\phi: \mathbb{D} \mapsto \Delta$ fixing 0 . The conformal radius of the Siegel disk $\Delta_{\theta}$ is the quantity

$$
r(\theta)=\left|\phi^{\prime}(0)\right| .
$$

For all other $\theta \in[0, \infty)$ we set $r(\theta)=0$.

By the Koebe One-Quarter Theorem of classical complex analysis, the internal radius of $\Delta_{\theta}$ is at least $r(\theta) / 4$. Yoccoz $[$ Yoc has shown that the sum

$$
\Phi(\theta)+\log r(\theta)
$$

is bounded from below independently of $\theta \in \mathcal{B}$. Recently, Buff and Chéritat have greatly improved this result by showing that:

Theorem 2.12 ([BC2]). The function $\theta \mapsto \Phi(\theta)+\log r(\theta)$ extends to $\mathbb{R}$ as a 1 periodic continuous function.

We remark that the following stronger conjecture exists (see [MMY):

Marmi-Moussa-Yoccoz Conjecture. MMY The function $\theta \mapsto \Phi(\theta)+$ $\log r(\theta)$ is Hölder of exponent $1 / 2$. 
Dependence of the conformal radius of a Siegel disk on the parameter. In this section we will show that the conformal radius of a Siegel disk varies continuously with the Julia set. To that end we will need a preliminary definition:

Definition 2.9. Let $\left(U_{n}, u_{n}\right)$ be a sequence of topological disks $U_{n} \subset \mathbb{C}$ with marked points $u_{n} \in U_{n}$. The kernel or Carathéodory convergence $\left(U_{n}, u_{n}\right) \rightarrow(U, u)$ means the following:

- $u_{n} \rightarrow u$;

- for any compact $K \subset U$ and for all $n$ sufficiently large, $K \subset U_{n}$;

- for any open connected set $W \ni u$, if $W \subset U_{n}$ for infinitely many $n$, then $W \subset U$.

The topology on the set of pointed domains which corresponds to the above definition of convergence is again called the kernel or Carathéodory topology. The meaning of this topology is as follows. For a pointed domain $(U, u)$ denote by

$$
\phi_{(U, u)}: \mathbb{D} \rightarrow U
$$

the unique conformal isomorphism with $\phi_{(U, u)}(0)=u$ and $\left(\phi_{(U, u)}\right)^{\prime}(0)>0$. We again denote by $r(U, u)=\left|\left(\phi_{(U, u)}\right)^{\prime}(0)\right|$ the conformal radius of $U$ with respect to $u$.

By the Riemann Mapping Theorem, the correspondence

$$
\iota:(U, u) \mapsto \phi_{(U, u)}
$$

establishes a bijection between marked topological disks properly contained in $\mathbb{C}$ and univalent maps $\phi: \mathbb{D} \rightarrow \mathbb{C}$ with $\phi^{\prime}(0)>0$. The following theorem is due to Carathéodory. A proof may be found in Pom.

Theorem 2.13 (Carathéodory Kernel Theorem). The mapping $\iota$ is a homeomorphism with respect to the Carathéodory topology on domains and the compactopen topology on maps.

Proposition 2.14. The conformal radius of a quadratic Siegel disk varies continuously with respect to the Hausdorff distance on Julia sets.

Proof. To fix the ideas, consider the family $P_{\theta}$ with $\theta \in \mathcal{B}$ and denote by $\Delta_{\theta}$ the Siegel disk of $P_{\theta}$. It is easy to see that the Hausdorff convergence $J\left(P_{\theta_{n}}\right) \rightarrow J\left(P_{\theta}\right)$ implies the Carathéodory convergence of the pointed domains

$$
\left(\Delta_{\theta_{n}}, 0\right) \rightarrow(\Delta, 0) \text {. }
$$

The proposition follows from this and the Carathéodory Kernel Theorem.

In fact, we can state the following quantitative version of the above result. For the proof, based on Koebe's Theorem, see e.g. [RZ].

Lemma 2.15. Let $U$ be a simply-connected bounded subdomain of $\mathbb{C}$ containing the point 0 in the interior. Suppose $V \subset U$ is a simply-connected subdomain of $U$, and $\partial V \subset U_{\epsilon}(\partial U)$. Then

$$
0<r(U, 0)-r(V, 0) \leq 4 \sqrt{r(U, 0)} \sqrt{\epsilon} .
$$

An immediate corollary is:

Corollary 2.16. Suppose the function $r(\theta)$ is non-computable on the set $\left\{\theta_{0}\right\}$. Then the function $\theta \mapsto J\left(P_{\theta}\right)$ is also non-computable at the same point. 
Proof. Assume that $J\left(P_{\theta_{0}}\right)$ is computable. Using the output of the TM computing this Julia set in an obvious way, for each $\epsilon>0$ we can obtain a domain $V \in \mathcal{C}$ such that

$$
V \subset \Delta_{\theta_{0}} \text { and } d_{H}\left(\partial V, \partial \Delta_{\theta_{0}}\right)<\epsilon .
$$

By Schwarz's Lemma, the conformal radius $r\left(\theta_{0}\right)<2$. Hence, by Lemma 2.15

$$
\left|r(V, 0)-r\left(\theta_{0}\right)\right|<\delta=8 \sqrt{\epsilon}
$$

Using any constructive version of the Riemann Mapping Theorem (see e.g. $[\mathrm{BB}]$ ), we can compute $r(V, 0)$ to precision $\delta$ and hence know $r\left(\theta_{0}\right)$ up to an error of $2 \delta$. Given that $\delta$ can be made arbitrarily small, we have shown that $r\left(\theta_{0}\right)$ is computable.

We also state for future reference the following proposition:

Proposition 2.17. Let $\left\{\theta_{i}\right\}$ be a sequence of Brjuno numbers such that $\theta_{i} \rightarrow \theta$ and $\varlimsup r\left(\theta_{i}\right)=l>0$. Then $\theta$ is also a Brjuno number and $r(\theta) \geq l$.

Proof. Let $\phi_{i} \equiv \phi_{\left(\Delta_{\theta_{i}}, 0\right)}$. Note that by Schwarz's Lemma, the inverse $\psi_{i} \equiv\left(\phi_{i}\right)^{-1}$ linearizes $P_{\theta_{i}}$ on $\Delta_{\theta_{i}}$. By passing to a subsequence we can assure that $\phi_{i} \rightarrow \phi$ locally uniformly, and $\phi^{\prime}(0) \geq l$. By continuity, $\phi^{-1}$ is a linearizing coordinate for $P_{\theta}$, so $\theta$ is a Brjuno number. Moreover, $\phi(\mathbb{D}) \subset \Delta_{\theta}$, and so by Schwarz's Lemma $r(\theta) \geq l$.

Non-computability of Yoccoz's Brjuno function. In addition to the noncomputability of the conformal radius, we also prove a non-computability result for Yoccoz's Brjuno function $\Phi$ :

Theorem 2.18 (Non-computability of $\Phi$ ). There exists a parameter value $\theta \in$ $\mathbb{R} / \mathbb{Z}$ such that $\Phi(\theta)<\infty$, and $\Phi(\theta)$ is not computable by any Turing Machine with an oracle for $\theta$.

It is worth noting that the Marmi-Moussa-Yoccoz Conjecture as stated above and Theorem 2.18 imply the existence of a non-computable quadratic Julia set. To see this, we first formulate:

Conditional Implication 2.19. If the Marmi-Moussa-Yoccoz Conjecture holds, then the function

$$
v: \theta \mapsto \Phi(\theta)+\log r(\theta)
$$

is computable by one Turing Machine on the entire interval $[0,1]$.

We use the following result of Buff and Chéritat ([BC2]).

Lemma 2.20 ([BC2 $)$. For any rational point $\theta=\frac{p}{q} \in[0,1]$ denote, as before,

$$
P_{\theta}(z)=e^{2 \pi i \theta} z+z^{2}
$$

and let the Taylor expansion of $P_{\theta}^{\circ q}(z)$ at 0 start with

$$
P_{\theta}^{\circ q}(z)=z+A z^{q+1}+\ldots, \text { for } q \in \mathbb{N} .
$$

Let $L(\theta)=\left(\frac{1}{q A}\right)^{1 / q}$. Denote by $\Phi_{\text {trunc }}$ the modification of $\Phi$ applied to rational numbers where the sum is truncated before the infinite term. Then we have the following explicit formula for computing $v(\theta)$ :

$$
v(\theta)=\Phi_{\text {trunc }}(\theta)+\log L(\theta)+\frac{\log 2 \pi}{q} .
$$


Equation (2.6) allows us to compute the value of $v$ easily at every rational $\theta \in$ $\mathbb{Q} \cap[0,1]$ with arbitrarily good precision. In addition, assuming the conjecture, we have $|v(x)-v(y)|<2^{-n}$ whenever $|x-y|<c \cdot 2^{-2 n}$ for some constant $c$; hence $v$ has an (easily) computable modulus of continuity. These two facts together imply that $v$ is computable by a single machine of the interval $[0,1]$ (see for example Proposition 2.6 in $\mathrm{Ko2}$ ). This implies the Conditional Implication.

The following conditional result follows:

Lemma 2.21 (Conditional). Suppose the Conditional Implication holds. Let $\theta \in[0,1]$ be such that $\Phi(\theta)$ is finite. Then there is an oracle Turing Machine $M_{1}^{\phi}$ computing $\Phi(\theta)$ with an oracle access to $\theta$ if and only if there is an oracle Turing Machine $M_{2}^{\phi}$ computing $r(\theta)$ with an oracle access to $\theta$.

Proof. Suppose that $M_{1}^{\phi}$ computes $\Phi(\theta)$ for some $\theta$. Let $M^{\phi}$ be the machine uniformly computing the function $v$. Then we can use $M_{1}^{\phi}$ and $M^{\phi}$ to compute $\log r(\theta)=v(\theta)-\Phi(\theta)$ with arbitrarily good precision. We can then use this construction to give a machine $M_{2}^{\phi}$ which computes $r(\theta)$.

The opposite direction is proved analogously.

Lemma 2.21 with Theorem 2.18 imply that there is a $\theta$ for which $r(\theta)$ is noncomputable. Corollary 2.16 implies that for this value of $\theta$ the Julia set of $P_{\theta}$ is also non-computable.

Note that for the proof of the Conditional Implication we did not need the full power of the conjecture. All we needed was some computable bound on the modulus of continuity of $v$.

Outline of the construction of a non-computable quadratic Julia set. We are now prepared to outline the idea of our construction. The outline given below is rather rough and suffers from obvious logical deficiencies. However, it captures the idea of the proof in a simple to understand form. Suppose that every Julia set of a polynomial $P_{\theta}$ is computable by an oracle machine $M^{\phi}$, where $\phi$ represents $\theta$. There are countably many machines, so we can enumerate them $M_{1}^{\phi}, M_{2}^{\phi}, \ldots$. Denote by $S_{i}$ the domain on which $M_{i}^{\phi}$ computes $J_{P_{\theta}}$ properly. Then we must have:

(1) $\mathbb{C}=\bigcup_{i=1}^{\infty} S_{i}$,

(2) for each $i$, the function $J: \theta \mapsto J\left(P_{\theta}\right)$ is continuous on $S_{i}$.

Let us start with a machine $M_{n_{1}}^{\phi}$ which computes $J\left(P_{\theta_{*}}\right)$ for $\theta_{*}=[1,1,1, \ldots]$. If any of the digits $r_{i}$ in this infinite continued fraction is changed to a sufficiently large $N \in \mathbb{N}$, the conformal radius of the Siegel disk will become small. For $N \rightarrow \infty$ the Siegel disk will implode and its center will become a parabolic fixed point in the Julia set. Given the continuity of the dependence of the conformal radius of the Siegel disk on the Julia set, we have the following:

There exists $i_{1}>1$ such that for every $\theta_{1}$ whose continued fraction starts with $i_{1}$ ones, for the Julia set of $P_{\theta_{1}}$ to be computable by $M_{n_{1}}^{\phi}$, it must possess a Siegel disk of a conformal radius $r\left(\theta_{1}\right)>r\left(\theta_{*}\right)(1-1 / 8)$.

We can thus "fool" the machine $M_{n_{1}}^{\phi}$ by selecting $\theta_{1}$ given by a continued fraction where all digits are ones except $r_{i_{1}}=N_{1}>>1$. If we are careful, we can do it so that

$$
r\left(\theta_{*}\right)(1-1 / 4)<r\left(\theta_{1}\right)<r\left(\theta_{*}\right)(1-1 / 8) .
$$




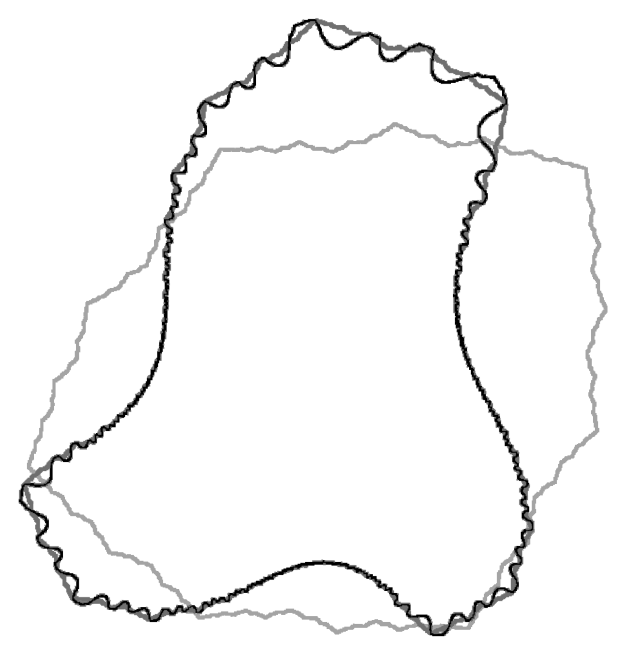

Figure 1. The Siegel disks of $P_{\theta}$ for $\theta$ given by the continued fractions $[1,1,1, \ldots], \quad[1,1,1,20,1, \ldots]$, and $[1,1,1,20,1,1,1$, $30,1, \ldots]$

To "fool" the machine $M_{n_{2}}^{\phi}$ we then change a digit $r_{i_{2}}$ for $i_{2}>i_{1}$ sufficiently far in the continued fraction of $\theta_{1}$ to a large $N_{2}$. In this way, we will obtain a Brjuno number $\theta_{2}$ for which

$$
r\left(\theta_{*}\right)(1-1 / 4-1 / 8)<r\left(\theta_{2}\right)<r\left(\theta_{*}\right)(1-1 / 4) .
$$

Continuing in this manner we will arrive at a limiting Brjuno number $\theta_{\infty}$ for which the Julia set is non-computable. To make such a scheme work, we need a careful analysis of the dependence of the conformal radius on the parameter. In this a key role is played by Theorem 2.12 of Buff and Chéritat, which allows us to obtain a controlled change in the value of $r(\alpha)$ by changing $\Phi(\alpha)$. The relevant analysis is carried out in the next section.

Main analytic result. We formalize the strategy outlined above as follows:

Theorem 2.22. There does not exist a partition of the circle $\mathbb{R} / \mathbb{Z}$ into a countable union of sets $S_{i}$ such that for every $i$ the function $r(\theta)$ restricted to $S_{i}$ is continuous.

The above formulation was suggested to us by John Milnor. Let us show how the Main Theorem and Theorem 2.18 follow from Theorem 2.22 .

Proof of Main Theorem, assuming Theorem 2.22. First we observe that there exists a parameter $\theta_{0} \in \mathbb{R} / \mathbb{Z}$ such that the function $r(\theta)$ is non-computable on $\left\{\theta_{0}\right\}$. Indeed, assume the contrary. There are only countably many Turing Machines with an oracle for $\theta$. We enumerate them $M_{i}, i \in \mathbb{N}$ in some arbitrary way (for instance, using the lexicographic order). Let

$$
S_{i}=\left\{\theta \in \mathbb{R} / \mathbb{Z} \text { such that TM } M_{i}^{\phi} \text { computes } r(\theta)\right\} .
$$

By Proposition 2.1 the function $r(\theta)$ is continuous on each of the $S_{i}$ 's, and we arrive at a contradiction with Theorem 2.22 .

Now let us prove the Main Theorem, again arguing by contradiction. Assume that for every $c \in \mathbb{C}$ there exists a TM $M^{\phi}$ with an oracle for $c$ which computes 
$J_{c}$. Let $P_{\theta}=z^{2}+e^{2 \pi i \theta} z$ as before. The affine change of coordinates transforming it into an element of the family $f_{c}$ is computable explicitly, and we have

$$
c=c(\theta)=\lambda / 2-\lambda^{2} / 4 \text { where } \lambda=e^{2 \pi i \theta} .
$$

This implies that we can simulate an oracle for $c$ given an oracle for $\theta$.

Set $c_{0}=c\left(\theta_{0}\right)$ and consider the oracle TM $M^{\phi}$ computing the Julia set of $f_{c_{0}}$. By the above considerations, there exists an oracle TM $\widetilde{M}^{\psi}$ with an oracle for $\theta \in \mathbb{R} / \mathbb{Z}$ which computes $J\left(P_{\theta_{0}}\right)$. This contradicts Corollary 2.16] and the proof is complete.

Proof of Theorem 2.18 assuming Theorem 2.22, Assume the contrary. Again, order in a sequence $M_{i}^{\phi}, i \in \mathbb{N}$ all TMs with an oracle for $\theta \in \mathbb{R} / \mathbb{Z}$. Let

$$
\Omega_{i}=\left\{\theta \in \mathbb{R} / \mathbb{Z} \text { such that } M_{i}^{\phi} \text { computes the value of } \Phi(\theta)\right\} .
$$

Denote by $\Omega_{0}$ the set of all $\theta$ with $\Phi(\theta)=\infty$. The value of $r(\theta)$ on $\Omega_{0}$ is thus identically 0 .

Let

$$
v(\theta)=\Phi(\theta)+\log r(\theta),
$$

which by [BC2] continuously extends to $\mathbb{R} / \mathbb{Z}$. Given Proposition 2.1, the function $\Phi(\theta)$ is continuous on each $\Omega_{i}, i \in \mathbb{N}$, and hence so is

$$
r(\theta)=\exp (v(\theta)-\Phi(\theta)) .
$$

By our assumption,

$$
\mathbb{R} / \mathbb{Z}=\bigcup_{i=0}^{\infty} \Omega_{i}
$$

and we arrive at a contradiction with Theorem 2.22

A note on the connection with $\mathrm{BC1}$. A. Chéritat has pointed out to us that the methods of [BC1, where Siegel disks with smooth boundaries are constructed for the quadratic family, can be used to derive the Main Theorem. We discuss this in the section following the proofs of the main theorems. We note here that the argument we give is based on quite elementary estimates of the function $\Phi$ and is thus accessible to non-dynamicists. It has an added advantage of yielding Theorem 2.18

\section{Making SMall Changes to $\Phi$}

3.1. Small changes to $\Phi$. A key step of the construction outlined above is making careful adjustments of $r\left(\theta_{i}\right)$ as in the first two steps (2.7) and (2.8) above. We do not have direct control over the value of $r(\alpha)$, but Buff and Chéritat's Theorem 2.12 shows that small decreases of $r(\alpha)$ we would like to make correspond to a small controlled increment of the value of $\Phi(\alpha)$. Estimates of a similar nature have appeared in the works of various authors (compare, for example, with [BC1]).

For a number $\gamma=\left[a_{1}, a_{2}, \ldots\right] \in \mathbb{R} \backslash \mathbb{Q}$ we denote

$$
\alpha_{i}(\gamma)=\frac{1}{a_{i}+\frac{1}{a_{i+1}+\frac{1}{a_{i+2}+\ldots}},},
$$


so that

$$
\Phi(\gamma)=\sum_{n \geq 1} \alpha_{1}(\gamma) \alpha_{2}(\gamma) \ldots \alpha_{n-1}(\gamma) \log \frac{1}{\alpha_{n}(\gamma)} .
$$

The main goal of this section is to prove the following two lemmas:

Lemma 3.1. For any initial segment $I=\left[a_{1}, a_{2}, \ldots, a_{n}\right]$, write $\omega=\left[a_{1}, a_{2}, \ldots, a_{n}\right.$, $1,1,1, \ldots]$. Then for any $\varepsilon>0$, there is an $m>0$ and an integer $N$ such that if we write $\beta^{N}=\left[a_{1}, a_{2}, \ldots, a_{n}, 1,1, \ldots, 1, N, 1,1, \ldots\right]$, where the $N$ is located in the $(n+m)$-th position, then

$$
\Phi(\omega)+\varepsilon<\Phi\left(\beta^{N}\right)<\Phi(\omega)+2 \varepsilon .
$$

Lemma 3.2. For $\omega$ as above, for any $\varepsilon>0$ there is an $m_{0}>0$, such that for any $m \geq m_{0}$, and for any tail $T=\left[a_{n+m}, a_{n+m+1}, \ldots\right]$, if we denote

$$
\beta^{T}=\left[a_{1}, a_{2}, \ldots, a_{n}, 1,1, \ldots, 1, a_{n+m}, a_{n+m+1}, \ldots\right],
$$

then

$$
\Phi\left(\beta^{T}\right)>\Phi(\omega)-\varepsilon .
$$

The proof is technical and will require some preparation. For Lemma 3.1 the idea is to choose an $m$ large enough, so that changing $a_{n+m}$ (which will eventually be $N$ ) by 1 changes the value of $\Phi$ by a very small amount $(<\varepsilon)$. When $N \rightarrow \infty$, $\Phi(\omega) \rightarrow \infty$; hence the value of $\Phi$ must hit the interval $(\Phi(\omega)+\varepsilon, \Phi(\omega)+2 \varepsilon)$.

Denote

$$
\Phi^{-}(\omega)=\Phi(\omega)-\alpha_{1} \alpha_{2} \ldots \alpha_{n+m-1} \log \frac{1}{\alpha_{m+n}} .
$$

The value of the integer $m>0$ is yet to be determined. Denote

$$
\beta^{N}=\left[a_{1}, a_{2}, \ldots, a_{n}, 1,1, \ldots, 1, N, 1,1, \ldots\right] .
$$

We prove the following.

Lemma 3.3. For any $N$ and $i \leq n+m$ we have

$$
\left|\log \frac{\alpha_{i}\left(\beta^{N}\right)}{\alpha_{i}\left(\beta^{N+1}\right)}\right|<\frac{2^{i-(n+m)}}{N} .
$$

Proof. We prove the lemma by induction on $i$, starting from the base case $i=n+m$, and proceeding down to $i=0$. The base case is $i=n+m$; we want to prove

$$
\left|\log \frac{\alpha_{n+m}\left(\beta^{N}\right)}{\alpha_{n+m}\left(\beta^{N+1}\right)}\right|<\frac{1}{N} \text {. }
$$

We have

$$
r_{n+m}=\frac{\alpha_{n+m}\left(\beta^{N}\right)}{\alpha_{n+m}\left(\beta^{N+1}\right)}=\frac{\frac{1}{N+1 / \phi}}{\frac{1}{N+1+1 / \phi}}=\frac{N+1+1 / \phi}{N+1 / \phi}=1+\frac{1}{N+1 / \phi},
$$

where $\phi=(\sqrt{5}+1) / 2$. Hence

$$
1<r_{n+m}<1+\frac{1}{N}<e^{1 / N},
$$

and $\left|\log r_{n+m}\right|<\frac{1}{N}$. 
Induction step. Supposing that the statement is true for $i+1$, we prove it for $i$. We have

$$
\frac{\alpha_{i}\left(\beta^{N}\right)}{\alpha_{i}\left(\beta^{N+1}\right)}=\frac{\frac{1}{a_{i}+\alpha_{i+1}\left(\beta^{N}\right)}}{\frac{1}{a_{i}+\alpha_{i+1}\left(\beta^{N+1}\right)}}=\frac{a_{i}+\alpha_{i+1}\left(\beta^{N+1}\right)}{a_{i}+\alpha_{i+1}\left(\beta^{N}\right)} .
$$

Suppose that $\alpha_{i+1}\left(\beta^{N+1}\right) \geq \alpha_{i+1}\left(\beta^{N}\right)$. Then we know that

$$
\frac{\alpha_{i+1}\left(\beta^{N+1}\right)}{\alpha_{i+1}\left(\beta^{N}\right)}<e^{2^{i+1-(n+m)} / N}
$$

and we want to prove that

$$
\frac{a_{i}+\alpha_{i+1}\left(\beta^{N+1}\right)}{a_{i}+\alpha_{i+1}\left(\beta^{N}\right)}<e^{2^{i-(n+m)} / N},
$$

since this expression is obviously bigger than 1 . The situation is very similar in the case when $\alpha_{i+1}\left(\beta^{N+1}\right) \leq \alpha_{i+1}\left(\beta^{N}\right)$, with the numerator and the denominator exchanged.

In other words, it is enough to prove that for $0<d<c<1$ and a pair of integers $r \geq 0, k \geq 1$ and $\alpha>0$,

$$
\frac{c}{d}<e^{\alpha} \text { implies that } \frac{k+c}{k+d}<e^{\alpha / 2} .
$$

First of all, it is easy to see that for $k \geq 1$,

$$
\frac{k+c}{k+d} \leq \frac{1+c}{1+d}
$$

hence it suffices to show that

$$
\frac{1+c}{1+d}<e^{\alpha / 2}=\left(e^{\alpha}\right)^{1 / 2}
$$

Thus we need to demonstrate that

$$
\frac{1+c}{1+d}<\left(\frac{c}{d}\right)^{1 / 2}
$$

This is equivalent to

$$
\left(1+2 c+c^{2}\right) d<\left(1+2 d+d^{2}\right) c \Leftrightarrow d+c^{2} d<c+d^{2} c \Leftrightarrow c d(c-d)<c-d .
$$

The last inequality holds, since $c d<1$ and $c-d>0$.

The following lemma is proven by induction exactly as the previous one with a different base.

Lemma 3.4. Let $\gamma_{1}$ and $\gamma_{2}$ be two numbers whose continued fraction expansions coincide in the first $n+m-1$ terms $\left[a_{1}, a_{2}, \ldots, a_{n+m-1}\right]$. Then for any $i<n+m$ we have

$$
\left|\log \frac{\alpha_{i}\left(\gamma_{1}\right)}{\alpha_{i}\left(\gamma_{2}\right)}\right|<2^{i-(n+m)+1}
$$

In particular, this applies with $\gamma_{1}=\beta^{N}$ and $\gamma_{2}=\beta^{1}$. 
Proof. The proof goes by induction exactly as in Lemma 3.3. We need to verify the base case $i=n+m-1$. For this value of $i$,

$$
\alpha_{n+m-1}\left(\gamma_{1}\right)=\frac{1}{a_{n+m-1}+\mu_{1}}, \alpha_{n+m-1}\left(\gamma_{2}\right)=\frac{1}{a_{n+m-1}+\mu_{2}},
$$

with some $\mu_{1}, \mu_{2} \in[0,1)$. Hence we have

$$
\left|\log \frac{\alpha_{n+m-1}\left(\gamma_{1}\right)}{\alpha_{n+m-1}\left(\gamma_{2}\right)}\right|<\log 2<1=2^{0} .
$$

We now bound the influence of the difference on the $\log \frac{1}{\alpha_{i}}$ terms.

Lemma 3.5. Let $\gamma_{1}$ and $\gamma_{2}$ be two numbers whose continued fraction expansions coincide in the first $n+m-1$ terms $\left[a_{1}, a_{2}, \ldots, a_{n+m-1}\right]$. Then for any $i<n+m-1$ we have

$$
\left|\log \frac{\log \frac{1}{\alpha_{i}\left(\gamma_{1}\right)}}{\log \frac{1}{\alpha_{i}\left(\gamma_{2}\right)}}\right|<2^{i-(n+m)+2} .
$$

In particular, this applies with $\gamma_{1}=\beta^{N}$ and $\gamma_{2}=\beta^{1}$.

Proof. Assume that $\alpha_{i}\left(\gamma_{1}\right) \leq \alpha_{i}\left(\gamma_{2}\right)$; the reverse case is done in the same way. In this case we need to prove

$$
\frac{\log \frac{1}{\alpha_{i}\left(\gamma_{1}\right)}}{\log \frac{1}{\alpha_{i}\left(\gamma_{2}\right)}}<e^{2^{i-(n+m)+2}} .
$$

Let $c=\alpha_{i+1}\left(\gamma_{1}\right)$ and $d=\alpha_{i+1}\left(\gamma_{2}\right)$. Then we have $\alpha_{i}\left(\gamma_{1}\right)=\frac{1}{k+c}$ and $\alpha_{i}\left(\gamma_{2}\right)=$ $\frac{1}{k+d}$ for some integer $k \geq 1$. Hence $0<d \leq c<1$. We have

$$
\frac{\log \frac{1}{\alpha_{i}\left(\gamma_{1}\right)}}{\log \frac{1}{\alpha_{i}\left(\gamma_{2}\right)}}=\frac{\log (k+c)}{\log (k+d)} .
$$

By Lemma 3.4 we know that $\frac{c}{d}<e^{2^{i-(n+m)+2}}$; hence it suffices to show that $\frac{\log (k+c)}{\log (k+d)} \leq \frac{c}{d}$. This is equivalent to $\frac{\log (k+c)}{c} \leq \frac{\log (k+d)}{d}$. Consider the function $f(x)=\frac{\log (k+x)}{x}$ on the interval $(0,1)$. The reader can readily verify that $f^{\prime}(x)<0$ for $x \stackrel{x}{\in}(0,1)$ so that $f$ is decreasing on this interval, and hence $f(c) \leq f(d)$, which completes the proof.

We are now ready to bound the influence of changes in $N$ on the value of $\Phi^{-}$.

Lemma 3.6. For any $\omega$ of the form as in Lemma 3.1 and for any $\varepsilon>0$, there is an $m_{0}>0$ such that for any $N$ and any $m \geq m_{0}$,

$$
\left|\Phi^{-}\left(\beta^{N}\right)-\Phi^{-}\left(\beta^{1}\right)\right|<\frac{\varepsilon}{4} .
$$


Proof. The $\sum$ in the expression for $\Phi\left(\beta^{1}\right)$ converges. Hence there is an $m_{1}>1$ such that the tail of the sum $\sum_{i \geq n+m_{1}} \alpha_{1} \alpha_{2} \ldots \alpha_{i-1} \log \frac{1}{\alpha_{i}}<\frac{\varepsilon}{40}$. We will show how to choose $m_{0}>m_{1}$ to satisfy the conclusion of the lemma.

We bound the influence of the change from $\beta^{1}$ to $\beta^{N}$ using Lemmas 3.4 and 3.5 . The influence on each of the "head elements" $\left(i<n+m_{1}\right)$ is bounded by

$$
\begin{aligned}
\left|\log \frac{\alpha_{1}\left(\beta^{1}\right) \ldots \alpha_{i-1}\left(\beta^{1}\right) \log \frac{1}{\alpha_{i}\left(\beta^{1}\right)}}{\alpha_{1}\left(\beta^{N}\right) \ldots \alpha_{i-1}\left(\beta^{N}\right) \log \frac{1}{\alpha_{i}\left(\beta^{N}\right)}}\right| & <\sum_{j=1}^{i-1} 2^{j-(n+m)+1}+2^{i-(n+m)+2} \\
& <2^{i-(n+m)+3}<2^{m_{1}+3-m} .
\end{aligned}
$$

By making $m$ sufficiently large (i.e., by choosing a sufficiently large $m_{0}$ ) we can ensure that

$$
1-\frac{\varepsilon}{40 \Phi\left(\beta^{1}\right)}<\frac{\alpha_{1}\left(\beta^{N}\right) \ldots \alpha_{i-1}\left(\beta^{N}\right) \log \frac{1}{\alpha_{i}\left(\beta^{N}\right)}}{\alpha_{1}\left(\beta^{1}\right) \ldots \alpha_{i-1}\left(\beta^{1}\right) \log \frac{1}{\alpha_{i}\left(\beta^{1}\right)}}<1+\frac{\varepsilon}{40 \Phi\left(\beta^{1}\right)} .
$$

Hence

$$
\begin{gathered}
\left|\alpha_{1}\left(\beta^{N}\right) \ldots \alpha_{i-1}\left(\beta^{N}\right) \log \frac{1}{\alpha_{i}\left(\beta^{N}\right)}-\alpha_{1}\left(\beta^{1}\right) \ldots \alpha_{i-1}\left(\beta^{1}\right) \log \frac{1}{\alpha_{i}\left(\beta^{1}\right)}\right| \\
<\frac{\varepsilon}{40 \Phi\left(\beta^{1}\right)} \alpha_{1}\left(\beta^{1}\right) \ldots \alpha_{i-1}\left(\beta^{1}\right) \log \frac{1}{\alpha_{i}\left(\beta^{1}\right)} .
\end{gathered}
$$

Adding the inequalities for $i=1,2, \ldots, n+m_{1}-1$ we obtain

$$
\begin{gathered}
\left|\sum_{i=1}^{n+m_{1}-1} \alpha_{1}\left(\beta^{N}\right) \ldots \alpha_{i-1}\left(\beta^{N}\right) \log \frac{1}{\alpha_{i}\left(\beta^{N}\right)}-\sum_{i=1}^{n+m_{1}-1} \alpha_{1}\left(\beta^{1}\right) \ldots \alpha_{i-1}\left(\beta^{1}\right) \log \frac{1}{\alpha_{i}\left(\beta^{1}\right)}\right| \\
\quad<\frac{\varepsilon}{40 \Phi\left(\beta^{1}\right)} \sum_{i=1}^{n+m_{1}-1} \alpha_{1}\left(\beta^{1}\right) \ldots \alpha_{i-1}\left(\beta^{1}\right) \log \frac{1}{\alpha_{i}\left(\beta^{1}\right)}<\frac{\varepsilon}{40 \Phi\left(\beta^{1}\right)} \Phi\left(\beta^{1}\right)=\frac{\varepsilon}{40} .
\end{gathered}
$$

Hence the influence on the "head" of $\Phi^{-}$is bounded by $\frac{\varepsilon}{40}$.

To bound the influence on the "tail" we consider three kinds of terms $\alpha_{1}\left(\beta^{N}\right) \ldots \alpha_{i-1}\left(\beta^{N}\right) \log \frac{1}{\alpha_{i}\left(\beta^{N}\right)}$ : those for which $n+m_{1} \leq i \leq n+m-2, i=$ $m+n-1$ and $i \geq m+n+1$ (recall that $i=n+m$ is not in $\Phi^{-}$).

- For $n+m_{1} \leq i \leq n+m-2$. By Lemmas 3.4 and 3.5 .

$$
\begin{aligned}
\left|\log \frac{\alpha_{1}\left(\beta^{1}\right) \ldots \alpha_{i-1}\left(\beta^{1}\right) \log \frac{1}{\alpha_{i}\left(\beta^{1}\right)}}{\alpha_{1}\left(\beta^{N}\right) \ldots \alpha_{i-1}\left(\beta^{N}\right) \log \frac{1}{\alpha_{i}\left(\beta^{N}\right)}}\right| & <\sum_{j=1}^{i-1} 2^{j-(n+m)+1}+2^{i-(n+m)+2} \\
& <2^{i-(n+m)+3} \leq 2 .
\end{aligned}
$$

Hence in this case each term can increase by a factor of at most $e^{2}$. 
- For $i=n+m-1$. Note that the change decreases $\log \frac{1}{\alpha_{n+m-1}}$ so that $\log \frac{1}{\alpha_{n+m-1}\left(\beta^{N}\right)} \leq \log \frac{1}{\alpha_{n+m-1}\left(\beta^{1}\right)}$. Hence we have

$$
\begin{gathered}
\log \frac{\alpha_{1}\left(\beta^{N}\right) \ldots \alpha_{i-1}\left(\beta^{N}\right) \log \frac{1}{\alpha_{i}\left(\beta^{N}\right)}}{\alpha_{1}\left(\beta^{1}\right) \ldots \alpha_{i-1}\left(\beta^{1}\right) \log \frac{1}{\alpha_{i}\left(\beta^{1}\right)}} \leq \log \frac{\alpha_{1}\left(\beta^{N}\right) \ldots \alpha_{i-1}\left(\beta^{N}\right)}{\alpha_{1}\left(\beta^{1}\right) \ldots \alpha_{i-1}\left(\beta^{1}\right)} \\
<\sum_{j=1}^{n+m-2} 2^{j-(n+m)+1}<1 .
\end{gathered}
$$

Hence this term could increase by a factor of at most $e$.

- For $i \geq n+m+1$. Note that the $\alpha_{j}$ for $j>n+m$ are not affected by the change, and the change decreases $\alpha_{n+m}$, so that $\alpha_{n+m}\left(\beta^{N}\right) \leq \alpha_{n+m}\left(\beta^{1}\right)$. Hence

$$
\begin{gathered}
\log \frac{\alpha_{1}\left(\beta^{N}\right) \ldots \alpha_{i-1}\left(\beta^{N}\right) \log \frac{1}{\alpha_{i}\left(\beta^{N}\right)}}{\alpha_{1}\left(\beta^{1}\right) \ldots \alpha_{i-1}\left(\beta^{1}\right) \log \frac{1}{\alpha_{i}\left(\beta^{1}\right)}}=\log \frac{\alpha_{1}\left(\beta^{N}\right) \ldots \alpha_{n+m}\left(\beta^{N}\right)}{\alpha_{1}\left(\beta^{1}\right) \ldots \alpha_{n+m}\left(\beta^{1}\right)} \\
\leq \log \frac{\alpha_{1}\left(\beta^{N}\right) \ldots \alpha_{n+m-1}\left(\beta^{N}\right)}{\alpha_{1}\left(\beta^{1}\right) \ldots \alpha_{n+m-1}\left(\beta^{1}\right)}<\sum_{j=1}^{n+m-1} 2^{j-(n+m)+1}<2 .
\end{gathered}
$$

So in this case each term could increase by a factor of at most $e^{2}$.

We see that after the change each term of the tail could increase by a factor of $e^{2}$ at most. The value of the tail remains positive in the interval $\left(0, \frac{e^{2} \varepsilon}{40}\right]$. Hence the change in the tail is bounded by $\frac{e^{2} \varepsilon}{40}<\frac{9 \varepsilon}{40}$.

So the total change in $\Phi^{-}$is bounded by

$$
\text { change in the "head" + change in the "tail" }<\frac{\varepsilon}{40}+\frac{9 \varepsilon}{40}=\frac{\varepsilon}{4} \text {. }
$$

The following lemma follows immediately from Lemma 3.6 .

Lemma 3.7. For any $\varepsilon$ and for the same $m_{0}(\varepsilon)$ as in Lemma 3.6, for any $m \geq m_{0}$ and $N$,

$$
\left|\Phi^{-}\left(\beta^{N}\right)-\Phi^{-}\left(\beta^{N+1}\right)\right|<\frac{\varepsilon}{2}
$$

Proof. We have

$\left|\Phi^{-}\left(\beta^{N}\right)-\Phi^{-}\left(\beta^{N+1}\right)\right| \leq\left|\Phi^{-}\left(\beta^{N}\right)-\Phi^{-}\left(\beta^{1}\right)\right|+\left|\Phi^{-}\left(\beta^{1}\right)-\Phi^{-}\left(\beta^{N+1}\right)\right|<\frac{\varepsilon}{4}+\frac{\varepsilon}{4}=\frac{\varepsilon}{2}$.

We will now have to take a closer look at the term $\alpha_{1} \ldots \alpha_{n+m-1} \log \frac{1}{\alpha_{m+m}}=$ $\Phi(\omega)-\Phi^{-}(\omega)$. We will need the following simple statement.

Lemma 3.8. For any $k>1, \alpha_{k-1} \alpha_{k}<\frac{1}{2}$. 
Proof. There is an integer $l \geq 1$ such that

$$
\alpha_{k-1} \alpha_{k}=\frac{1}{l+\alpha_{k}} \alpha_{k}<\frac{1}{\alpha_{k}+\alpha_{k}} \alpha_{k}=\frac{1}{2} .
$$

Letting $\Phi^{1}(\alpha)=\alpha_{1} \ldots \alpha_{n+m-1} \log \frac{1}{\alpha_{n+m}}=\Phi(\omega)-\Phi^{-}(\omega)$, we are now ready to prove the following.

Lemma 3.9. For sufficiently large $m$, for any $N$,

$$
\Phi^{1}\left(\beta^{N+1}\right)-\Phi^{1}\left(\beta^{N}\right)<\frac{\varepsilon}{2} .
$$

Proof. According to Lemma 3.3 we have

$$
\left|\log \frac{\alpha_{1}\left(\beta^{N+1}\right) \ldots \alpha_{n+m-1}\left(\beta^{N+1}\right)}{\alpha_{1}\left(\beta^{N}\right) \ldots \alpha_{n+m-1}\left(\beta^{N}\right)}\right|<\sum_{i=1}^{n+m-1} 2^{i-(n+m)} / N<\frac{1}{N} .
$$

Hence $\alpha_{1}\left(\beta^{N+1}\right) \ldots \alpha_{n+m-1}\left(\beta^{N+1}\right)<\alpha_{1}\left(\beta^{N}\right) \ldots \alpha_{n+m-1}\left(\beta^{N}\right) e^{1 / N}$, and

$$
\Phi^{1}\left(\beta^{N+1}\right)<\Phi^{1}\left(\beta^{N}\right) e^{1 / N} \frac{\log \frac{1}{\alpha_{n+m}\left(\beta^{N+1}\right)}}{\log \frac{1}{\alpha_{n+m}\left(\beta^{N}\right)}}=\Phi^{1}\left(\beta^{N}\right) e^{1 / N} \frac{\log (N+1+1 / \phi)}{\log (N+1 / \phi)} .
$$

Hence

$$
\begin{gathered}
\Phi^{1}\left(\beta^{N+1}\right)-\Phi^{1}\left(\beta^{N}\right)<\Phi^{1}\left(\beta^{N}\right)\left(e^{1 / N} \frac{\log (N+1+1 / \phi)}{\log (N+1 / \phi)}-1\right) \\
<\Phi^{1}\left(\beta^{N}\right)\left(\left(1+\frac{e}{N}\right) \frac{\log (N+1+1 / \phi)}{\log (N+1 / \phi)}-1\right) .
\end{gathered}
$$

We make the following calculations. Let $x=\frac{\log (N+1+1 / \phi)}{\log (N+1 / \phi)}$. Then $(N+1 / \phi)^{x}=$ $N+1+1 / \phi$, and

$$
(N+1 / \phi)^{x-1}=\frac{N+1+1 / \phi}{N+1 / \phi}=1+\frac{1}{N+1 / \phi}<e^{\frac{1}{N+1 / \phi}} .
$$

$N+1 / \phi>e^{1 / 3}$, and so $x-1<\frac{3}{N+1 / \phi}<\frac{3}{N} ;$ thus $x<1+\frac{3}{N}$.

By Lemma 3.8 we have

$$
\Phi^{1}\left(\beta^{N}\right)=\alpha_{1}\left(\beta^{N}\right) \ldots \alpha_{n+m-1}\left(\beta^{N}\right) \log \frac{1}{\alpha_{n+m}\left(\beta^{N}\right)}<\left(\frac{1}{2}\right)^{(n+m-2) / 2} \log (N+1 / \phi) .
$$

Thus

$$
\begin{aligned}
& \Phi^{1}\left(\beta^{N+1}\right)-\Phi^{1}\left(\beta^{N}\right)<\Phi^{1}\left(\beta^{N}\right)\left(\left(1+\frac{e}{N}\right) \frac{\log (N+1+1 / \phi)}{\log (N+1 / \phi)}-1\right) \\
& <\left(\frac{1}{2}\right)^{(n+m-2) / 2} \log (N+1 / \phi)((1+e / N)(1+3 / N)-1) \\
& <\left(\frac{1}{2}\right)^{(n+m-2) / 2} \log (N+1 / \phi) \frac{14}{N} .
\end{aligned}
$$


Since $\frac{14}{N} \in o(1 / \log (N+1 / \phi))$, this expression can always be made less than $\frac{\varepsilon}{2}$ by choosing $m$ large enough.

Lemmas 3.7 and 3.9 yield the following.

Lemma 3.10. For sufficiently large $m$, for any $N$,

$$
\Phi\left(\beta^{N+1}\right)-\Phi\left(\beta^{N}\right)<\varepsilon .
$$

Proof. We use Lemmas 3.7 and 3.9 . For sufficiently large $m$,

$$
\Phi\left(\beta^{N+1}\right)-\Phi\left(\beta^{N}\right) \leq \Phi^{-}\left(\beta^{N+1}\right)-\Phi^{-}\left(\beta^{N}\right)+\Phi^{1}\left(\beta^{N+1}\right)-\Phi^{1}\left(\beta^{N}\right)<\frac{\varepsilon}{2}+\frac{\varepsilon}{2}=\varepsilon .
$$

To complete the proof of Lemma 3.1 we will need the following statement.

\section{Lemma 3.11.}

$$
\lim _{N \rightarrow \infty} \Phi\left(\beta^{N}\right)=\infty
$$

Proof. We will prove that $\lim _{N \rightarrow \infty} \Phi^{1}\left(\beta^{N}\right)=\infty$. This suffices since $\Phi^{1}\left(\beta^{N}\right)<$ $\Phi\left(\beta^{N}\right)$. By Lemma 3.4 ,

$$
\left|\log \frac{\alpha_{1}\left(\beta^{N}\right) \ldots \alpha_{n+m-1}\left(\beta^{N}\right)}{\alpha_{1}\left(\beta^{1}\right) \ldots \alpha_{n+m-1}\left(\beta^{1}\right)}\right|<\sum_{i=1}^{n+m-1} 2^{i-(n+m)+1}<2 .
$$

Hence

$$
\alpha_{1}\left(\beta^{N}\right) \ldots \alpha_{n+m-1}\left(\beta^{N}\right)>\frac{1}{e^{2}} \cdot \alpha_{1}\left(\beta^{1}\right) \ldots \alpha_{n+m-1}\left(\beta^{1}\right)
$$

and

$$
\Phi^{1}\left(\beta^{N}\right)>\frac{1}{e^{2}} \cdot \frac{\log (N+1 / \phi)}{\log (1+1 / \phi)} \Phi^{1}\left(\beta^{1}\right) .
$$

The latter expression obviously goes to $\infty$ as $N \rightarrow \infty$.

We are now ready to prove Lemma 3.1 .

Proof of Lemma 3.1. Choose $m$ large enough for Lemma 3.10 to hold. Increase $N$ by one at a time starting with $N=1$. We know that $\Phi\left(\beta^{1}\right)=\Phi(\omega)<\Phi(\omega)+\varepsilon$, and by Lemma 3.11, there exists an $M$ with $\Phi\left(\beta^{M}\right)>\Phi(\omega)+\varepsilon$. Let $N$ be the smallest such $M$. Then $\Phi\left(\beta^{N-1}\right) \leq \Phi(\omega)+\varepsilon$, and by Lemma 3.10 .

$$
\Phi\left(\beta^{N}\right)<\Phi\left(\beta^{N-1}\right)+\varepsilon \leq \Phi(\omega)+2 \varepsilon .
$$

Hence

$$
\Phi(\omega)+\varepsilon<\Phi\left(\beta^{N}\right)<\Phi(\omega)+2 \varepsilon .
$$

Choosing $\beta=\beta^{N}$ completes the proof.

We will now prove Lemma 3.2

Proof of Lemma 3.2. The $\sum$ in the expression for $\Phi(\omega)$ converges. Hence there is an $m_{1}>1$ such that the tail of the sum $\sum_{i \geq n+m_{1}} \alpha_{1} \alpha_{2} \ldots \alpha_{i-1} \log \frac{1}{\alpha_{i}}<\frac{\varepsilon}{2}$. We will show how to choose $m_{0}>m_{1}$ to satisfy the conclusion of the lemma. 
By Lemmas 3.4 and 3.5. for any $\beta^{T}$ and any $i \leq n+m_{1}$ we have

$$
\begin{gathered}
\left|\log \frac{\alpha_{1}\left(\beta^{T}\right) \ldots \alpha_{i-1}\left(\beta^{T}\right) \log \frac{1}{\alpha_{i}\left(\beta^{T}\right)}}{\alpha_{1}(\omega) \ldots \alpha_{i-1}(\omega) \log \frac{1}{\alpha_{i}(\omega)}}\right|<\sum_{j=1}^{i-1} 2^{j-(n+m)+1}+2^{i-(n+m)+2} \\
<2^{i-(n+m)+3} \leq 2^{n+m_{1}-\left(n+m_{0}\right)+3}=2^{m_{1}-m_{0}+3} .
\end{gathered}
$$

We can choose $m_{0}$ sufficiently large so that $e^{-2^{m_{1}-m_{0}+3}}>1-\frac{\varepsilon}{2 \Phi(\omega)}$ and so that

$$
\alpha_{1}\left(\beta^{T}\right) \ldots \alpha_{i-1}\left(\beta^{T}\right) \log \frac{1}{\alpha_{i}\left(\beta^{T}\right)}>\left(1-\frac{\varepsilon}{2 \Phi(\omega)}\right) \alpha_{1}(\omega) \ldots \alpha_{i-1}(\omega) \log \frac{1}{\alpha_{i}(\omega)},
$$

for $i \leq n+m_{1}$.

Now, for any $\beta^{T}$ we have

$$
\begin{gathered}
\Phi\left(\beta^{T}\right) \geq \sum_{i=1}^{n+m_{1}-1} \alpha_{1}\left(\beta^{T}\right) \alpha_{2}\left(\beta^{T}\right) \ldots \alpha_{i-1}\left(\beta^{T}\right) \log \frac{1}{\alpha_{i}\left(\beta^{T}\right)} \\
>\sum_{i=1}^{n+m_{1}-1}\left(1-\frac{\varepsilon}{2 \Phi(\omega)}\right) \alpha_{1}(\omega) \ldots \alpha_{i-1}(\omega) \log \frac{1}{\alpha_{i}(\omega)} \\
=\left(1-\frac{\varepsilon}{2 \Phi(\omega)}\right)\left(\Phi(\omega)-\sum_{i=n+m_{1}}^{\infty} \alpha_{1}(\omega) \ldots \alpha_{i-1}(\omega) \log \frac{1}{\alpha_{i}(\omega)}\right) \\
>\left(1-\frac{\varepsilon}{2 \Phi(\omega)}\right)\left(\Phi(\omega)-\frac{\varepsilon}{2}\right)>\Phi(\omega)-\varepsilon .
\end{gathered}
$$

We will also need the following lemma in the proof of the Main Theorem.

Lemma 3.12. Let $\omega=\left[a_{1}, a_{2}, a_{3}, \ldots\right]$ and let $\varepsilon>0$ be given. Then there is an $N=N(\varepsilon)$ such that for any $n \geq N$ we have $\Phi\left(\omega_{n}\right)<\Phi(\omega)+\varepsilon$, where $\omega_{n}=$ $\left[a_{1}, a_{2}, \ldots, a_{n}, 1,1,1, \ldots\right]$.

The proof is not hard and is similar to the proof of Lemma 3.6. We present the main steps in the proof.

- There is an $m_{0}$ such that the sum of the tail elements of $\Phi(\omega)$ is small:

$$
\sum_{i=m_{0}}^{\infty} \alpha_{1}(\omega) \ldots \alpha_{i-1}(\omega) \log \frac{1}{\alpha_{i}(\omega)}<\frac{\varepsilon}{4 \cdot e^{2}} .
$$

- Similarly to Lemma 3.6. we can use Lemmas 3.4 and 3.5 to show that for sufficiently large $m_{1}>m_{0}, n>m_{1}$ implies that

$$
\frac{\alpha_{1}\left(\omega_{n}\right) \ldots \alpha_{i-1}\left(\omega_{n}\right) \log \frac{1}{\alpha_{i}\left(\omega_{n}\right)}}{\alpha_{1}(\omega) \ldots \alpha_{i-1}(\omega) \log \frac{1}{\alpha_{i}(\omega)}}<1+\frac{\varepsilon}{4 \Phi(\omega)}
$$

for all $i<m_{0}$.

- Again by Lemmas 3.4, 3.5 and 3.8 we can show that for any $i$ (with a special consideration to the case $i=n$ ),

$$
\alpha_{1}\left(\omega_{n}\right) \ldots \alpha_{i-1}\left(\omega_{n}\right) \log \frac{1}{\alpha_{i}\left(\omega_{n}\right)}<\max \left(e^{2} \cdot \alpha_{1}(\omega) \ldots \alpha_{i-1}(\omega) \log \frac{1}{\alpha_{i}(\omega)}, 2^{2-i / 2}\right) .
$$


Adding these up we get for $n>m_{1}$ :

$$
\begin{gathered}
\Phi\left(\omega_{n}\right)<\left(1+\frac{\varepsilon}{4 \Phi(\omega)}\right) \sum_{i=1}^{m_{0}-1} \alpha_{1}(\omega) \ldots \alpha_{i-1}(\omega) \log \frac{1}{\alpha_{i}(\omega)} \\
+\sum_{i=m_{0}}^{\infty} \max \left(e^{2} \cdot \alpha_{1}(\omega) \ldots \alpha_{i-1}(\omega) \log \frac{1}{\alpha_{i}(\omega)}, 2^{2-i / 2}\right) \\
\leq\left(1+\frac{\varepsilon}{4 \Phi(\omega)}\right) \Phi(\omega)+\sum_{i=m_{0}}^{\infty} e^{2} \cdot \alpha_{1}(\omega) \ldots \alpha_{i-1}(\omega) \log \frac{1}{\alpha_{i}(\omega)}+\sum_{i=m_{0}}^{\infty} 2^{2-i / 2} \\
\leq \Phi(\omega)+\frac{\varepsilon}{4}+\frac{e^{2} \cdot \varepsilon}{4 \cdot e^{2}}+2^{4-m_{0} / 2}=\Phi(\omega)+\frac{\varepsilon}{2}+2^{4-m_{0} / 2} .
\end{gathered}
$$

We complete the proof by choosing $m_{0}$ large enough so that $2^{4-m_{0} / 2}<\varepsilon / 2$.

\section{Proof of Theorem 2.22}

Recall that $r(\theta)$ denotes the conformal radius of the Siegel disk associated with the polynomial $P_{\theta}(z)=z^{2}+e^{2 \pi i \theta} z$, or zero, if $\theta$ is not a Brjuno number.

We will argue by way of contradiction, and assume that there exists a countable union of sets

$$
\bigcup_{i=1}^{\infty} S_{i}=\mathbb{R} / \mathbb{Z}
$$

such that the function $r(\theta)$ is continuous on each $S_{i}$.

Main Lemma 4.1. There exist

- a sequence of initial segments $I_{i}=\left[a_{1}, a_{2}, \ldots, a_{N_{i}}\right]$, and

- a sequence of nested intervals

$$
\left[l_{0}, r_{0}\right] \supset\left[l_{1}, r_{1}\right] \supset\left[l_{2}, r_{2}\right] \supset \ldots,
$$

such that the following properties are maintained:

(1) whenever $i>j$ we have

$$
I_{i}=\left[I_{j}, a_{N_{j}+1}, a_{N_{j}+2}, \ldots, a_{N_{i}}\right] ;
$$

(2) $r_{i}=r\left(\gamma_{i}\right)$, where $\gamma_{i}=\left[I_{i}, 1,1, \ldots\right]$;

(3) for each $i \geq 1$ and for every $\beta=\left[I_{i}, t_{N_{i}+1}, t_{N_{i}+2}, \ldots\right]$ with $r(\beta) \in\left[l_{i}, r_{i}\right]$ we have

$$
\beta \notin S_{i}
$$

(4) denoting $\ell_{i}=r_{i}-l_{i}$

$$
\ell_{i}>0 \text { and } \ell_{i} \leq \ell_{i-1} / 2 \text { for all } i \geq 1
$$

(5) for any $\beta=\left[I_{i}, t_{N_{i}+1}, t_{N_{i}+2}, \ldots\right], i \geq 1$, we have

$$
\Phi(\beta)>\Phi\left(\gamma_{i-1}\right)-2^{-(i-1)} .
$$

Proof of the Main Lemma. We prove the Main Lemma by induction on $i$. For the basis of induction, set $I_{0}=[1], r_{0}=r\left(\gamma_{0}\right)$ and $l_{0}=r_{0} / 2$, where $\gamma_{0}=[1,1,1, \ldots]$. Then for $i=0$ conditions (1)-(5) trivially hold. 
The induction step. We now have the conditions satisfied for some $i \geq 0$ and would like to extend them to $i+1$.

Set $S \equiv S_{i+1}$. There are two possibilities:

Case 1. There exist $\varepsilon_{0}>0$ and $m_{0} \in \mathbb{N}$ such that for every $\beta \in S$ of the form

$$
\beta=[I_{i}, \underbrace{1,1, \ldots, 1}_{m_{0}}, \ldots] \text { we have }\left|r_{i}-r(\beta)\right|>\varepsilon_{0} .
$$

In this case, select $0<\varepsilon \leq \min \left(\varepsilon_{0}, \ell_{i} / 2\right)$. Set

$$
I_{i+1}=[I_{i}, \underbrace{1,1, \ldots, 1}_{m_{0}}], l_{i+1}=r_{i}-\varepsilon, \text { and } r_{i+1}=r_{i} .
$$

$\gamma_{i+1}=\left[I_{i}, 1,1, \ldots\right]=\gamma_{i}$. We have $r\left(\gamma_{i+1}\right)=r\left(\gamma_{i}\right)=r_{i}=r_{i+1}$. Hence conditions (11), (2) and (4) are satisfied.

Suppose $\beta=\left[I_{i+1}, t_{N_{i+1}+1}, t_{N_{i+1}+2}, \ldots\right]$ with $r(\beta) \in\left[l_{i+1}, r_{i+1}\right]$. Then $\beta \notin S$ and (3) is satisfied. By Lemma 3.2, we can choose $m_{0}$ sufficiently large in $I_{i+1}$, so that for any $\beta$ beginning with $I_{i+1}$, we have $\Phi(\beta)>\Phi\left(\gamma_{i}\right)-2^{-i}$, thus satisfying (5).

The complementary case is the main part of the argument:

Case 2. For every $\varepsilon>0$ and $m \in \mathbb{N}$ we can find $\beta \in S$ starting with $I_{i}$ followed by $m$ ones so that

$$
r_{i}-\varepsilon<r(\beta) \leq r_{i} .
$$

Choose an $\varepsilon$ such that $r_{i}-3 \varepsilon>l_{i}>r_{i}-4 \varepsilon$. Denote

$$
\varepsilon_{0}=\min \left(\frac{\log \left(r_{i}-\varepsilon\right)-\log \left(r_{i}-2 \varepsilon\right)}{8}, \frac{\log \left(r_{i}-2 \varepsilon\right)-\log \left(r_{i}-3 \varepsilon\right)}{8}\right)>0 .
$$

Theorem 2.12 of Buff and Chéritat says that the function

$$
v: \theta \mapsto \Phi(\theta)+\log r(\theta)
$$

continuously extends to $\mathbb{R} / \mathbb{Z}$. Due to compactness of $\mathbb{R} / \mathbb{Z}$, this function is uniformly continuous, and there exists a $\delta_{0}>0$ such that if $|x-y|<\delta_{0}$, then $|v(x)-v(y)|<\varepsilon_{0}$.

We choose $m$ large enough, so that for any $\zeta=[I_{i}, \underbrace{1,1, \ldots, 1}_{m}, \ldots]$ we have $\left|\gamma_{i}-\zeta\right|<\delta_{0}$ and so that Lemma 3.2 holds for $m_{0}=m$ with $I=I_{i}$ and $\varepsilon=2^{-i}$. Write

$$
\beta=[I_{i}, \underbrace{1,1, \ldots, 1}_{m}, t_{N_{i}+m+1}, t_{N_{i}+m+2}, \ldots] \in S .
$$

By assumption, the conformal radius $r(\bullet)$ is continuous on $S$. Hence there is a $\delta>0$ such that

$$
|r(x)-r(\beta)|<\varepsilon \text { whenever }|x-\beta|<\delta \text { and } x \in S .
$$

By Lemma 3.12, there is an $N$ such that for any $n \geq N$,

$$
\beta_{n}=[I_{i}, \underbrace{1,1, \ldots, 1}_{m}, t_{N_{i}+m+1}, \ldots, t_{N_{i}+m+n}, 1,1, \ldots]
$$

satisfies

$$
\Phi\left(\beta_{n}\right)<\Phi(\beta)+\varepsilon_{0}
$$


We can choose $n \geq N$ large enough so that for any $x$ whose continued fraction expansion has the initial segment

$$
I_{i}^{0}=[I_{i}, \underbrace{1,1, \ldots, 1}_{m}, t_{N_{i}+m+1}, t_{N_{i}+m+2}, \ldots, t_{N_{i}+m+n}],
$$

we have

$$
|x-\beta|<\min \left(\delta_{0} / 2, \delta\right) .
$$

Start with $\omega_{0}=\beta_{n}=\left[I_{i}^{0}, 1,1, \ldots\right]$. We have $\left|\omega_{0}-\beta\right|<\delta_{0}$, and hence $\mid v\left(\omega_{0}\right)-$ $v(\beta) \mid<\varepsilon_{0}$. So

$$
\log r\left(\omega_{0}\right)=v\left(\omega_{0}\right)-\Phi\left(\omega_{0}\right)>v(\beta)-\varepsilon_{0}-\Phi(\beta)-\varepsilon_{0}=\log r(\beta)-2 \varepsilon_{0} .
$$

By Lemma 3.1 we can extend $I_{i}^{0}$ to a longer initial segment $I_{i}^{1}$ so that setting $\omega_{1}=\left[I_{i}^{1}, 1,1, \ldots\right]$ we have

$$
\Phi\left(\omega_{0}\right)+2 \varepsilon_{0}<\Phi\left(\omega_{1}\right)<\Phi\left(\omega_{0}\right)+4 \varepsilon_{0} .
$$

We have $\left|\omega_{0}-\beta\right|<\delta_{0} / 2$ and $\left|\omega_{1}-\beta\right|<\delta_{0} / 2$, so $\left|\omega_{0}-\omega_{1}\right|<\delta_{0}$, and $\mid v\left(\omega_{0}\right)-$ $v\left(\omega_{1}\right) \mid<\varepsilon_{0}$. Hence

$$
\log \left(r\left(\omega_{1}\right)\right)=v\left(\omega_{1}\right)-\Phi\left(\omega_{1}\right)>v\left(\omega_{0}\right)-\varepsilon_{0}-\Phi\left(\omega_{0}\right)-4 \varepsilon_{0}=\log \left(r\left(\omega_{0}\right)\right)-5 \varepsilon_{0}
$$

and

$$
\log \left(r\left(\omega_{1}\right)\right)=v\left(\omega_{1}\right)-\Phi\left(\omega_{1}\right)<v\left(\omega_{0}\right)+\varepsilon_{0}-\Phi\left(\omega_{0}\right)-2 \varepsilon_{0}=\log \left(r\left(\omega_{0}\right)\right)-\varepsilon_{0} .
$$

Hence

$$
\log \left(r\left(\omega_{0}\right)\right)-5 \varepsilon_{0}<\log \left(r\left(\omega_{1}\right)\right)<\log \left(r\left(\omega_{0}\right)\right)-\varepsilon_{0} .
$$

In the same fashion, we can extend $I_{i}^{1}$ to $I_{i}^{2}$ and obtain $\omega_{2}=\left[I_{i}^{2}, 1,1, \ldots\right]$ so that

$$
\log \left(r\left(\omega_{1}\right)\right)-5 \varepsilon_{0}<\log \left(r\left(\omega_{2}\right)\right)<\log \left(r\left(\omega_{1}\right)\right)-\varepsilon_{0} .
$$

Recall that

$$
\log \left(r\left(\omega_{0}\right)\right)>\log (r(\beta))-2 \varepsilon_{0}>\log \left(r_{i}-\varepsilon\right)-2 \varepsilon_{0} \geq \log \left(r_{i}-2 \varepsilon\right)+6 \varepsilon_{0} .
$$

Hence, after finitely many steps, we will obtain $I_{i}^{k}$ and $\omega_{k}=\left[I_{i}^{k}, 1,1, \ldots\right]$ such that

$$
\log \left(r_{i}-3 \varepsilon\right)+\varepsilon_{0}<\log \left(r\left(\omega_{k}\right)\right)<\log \left(r_{i}-3 \varepsilon\right)+6 \varepsilon_{0}<\log \left(r_{i}-2 \varepsilon\right) .
$$

Choose $I_{i+1}=I_{i}^{k}, \gamma_{i+1}=\omega_{k}, l_{i+1}=l_{i}$, and $r_{i+1}=r\left(\omega_{k}\right)$. We have

$$
l_{i+1}<r_{i}-3 \varepsilon<r_{i+1}<r_{i}-2 \varepsilon .
$$

Conditions (11) and (2) are satisfied by definition. Condition (4) is satisfied because

$$
\ell_{i}=r_{i}-l_{i}<4 \varepsilon \text { and } \ell_{i+1}<\ell_{i}-2 \varepsilon<\ell_{i} / 2 .
$$

Condition (3) is satisfied, because $x=\left[I_{i}^{0}, \ldots\right] \in S$ only when $r(x) \in[r(\beta)-$ $\varepsilon, r(\beta)+\varepsilon] \subset\left[r_{i}-2 \varepsilon, r_{i}+\varepsilon\right]$, and by our construction $\left[l_{i+1}, r_{i+1}\right]$ is disjoint from $\left[r_{i}-2 \varepsilon, r_{i}+\varepsilon\right]$. Finally, condition (5) is satisfied because Lemma 3.2 holds with $\varepsilon=2^{-i}$ with the $m$ chosen in the initial segment $I_{i}^{0}$. We have thus completed the proof of the Main Lemma 4.1 . 
Passing to the limit. The completion of the proof of Theorem 2.22 relies on the following lemma:

Lemma 4.2. Denote $\gamma=\lim _{i \rightarrow \infty} \gamma_{i}$. Then the following equalities hold:

$$
\Phi(\gamma)=\lim _{i \rightarrow \infty} \Phi\left(\gamma_{i}\right) \text { and } r(\gamma)=\lim _{i \rightarrow \infty} r\left(\gamma_{i}\right) .
$$

Proof. By the construction, the $\operatorname{limit} \gamma=\lim \gamma_{i}$ exists. We also know by condition (41) of the Main Lemma4.1 that the sequence $r\left(\gamma_{i}\right)=r_{i}$ converges uniformly to some number $r$. By condition (5) of the Main Lemma 4.1 the sequence $\Phi\left(\gamma_{i}\right)-2^{-(i-1)}$ is non-decreasing and hence converges to a value $\psi$ (a priori we could have $\psi=\infty$ ). The sequence $\Phi\left(\gamma_{i}\right)$ must converge to $\psi$ as well.

By Proposition 2.17, we have $r(\gamma) \geq r>0$. On the other hand, by condition (51) of the Main Lemma 4.1, we know that $\Phi(\gamma)>\Phi\left(\gamma_{i}\right)-2^{-(i-1)}$ for all $i$. Hence $\Phi(\gamma) \geq \psi$. In particular $\psi<\infty$.

From [BC2] we know that

$$
\psi+\log r=\lim \left(\Phi\left(\gamma_{i}\right)+\log r\left(\gamma_{i}\right)\right)=\Phi(\gamma)+\log r(\gamma) .
$$

Along with $r(\gamma) \geq r$ and $\Phi(\gamma) \geq \psi$ this yields $\Phi(\gamma)=\psi$, and $r(\gamma)=r$, which completes the proof.

Finalizing the argument. Let $\gamma$ be the limit from the previous lemma. We claim that $\gamma \notin \bigcup S_{i}$. Indeed, for every $i$, the continued fraction $I_{i}$ is an initial segment of the continued fraction expansion of $\gamma$ by condition (1) of the Main Lemma 4.1. By Lemma 4.2 .

$$
r(\gamma)=\lim r\left(\gamma_{i}\right)=\lim r_{i} \in\left[l_{i}, r_{i}\right]
$$

Thus by condition (3) of the Main Lemma 4.1 we have $\gamma \notin S_{i}$. We have in this way arrived at a contradiction with $\bigcup S_{i}=\mathbb{R} / \mathbb{Z}$, which completes the proof of Theorem 2.22

\section{Concluding Remarks}

Connection with the work of Buff and Chéritat. Let us outline here how the methods of [BC1] can be applied to prove Theorem 2.22 instead of the estimates of 33 (we note that a newer version of the same result exists $\mathrm{ABC}$, where the arguments we quote are simplified). The main technical result of that paper is the following. Let $\alpha=\left[a_{0}, a_{1}, \ldots\right]$ be a Brjuno number, and as before denote by $p_{k} / q_{k}$ the sequence of its continued fraction approximants. Let $A>1$ and for each integer $n \geq 0$ set

$$
\alpha[n]=\left[a_{0}, a_{1}, \ldots, a_{n}, A^{q_{n}}, 1,1,1, \ldots\right] .
$$

Then for this particular sequence of Brjuno approximants of $\alpha$,

$$
\Phi(\alpha[n]) \underset{n \rightarrow \infty}{\longrightarrow} \Phi(\alpha)+\log A,
$$

and moreover,

$$
\lim r(\alpha[n])=r(\alpha) / A
$$

The last equality can be used to construct the "drops" in the value of the conformal radius of the Siegel disk needed to inductively avoid the sets $S_{i}$. In this way, one obtains a sequence of Brjuno numbers $\theta_{i} \rightarrow \theta$ with conformal radii $r_{i}=r\left(\theta_{i}\right)>r_{i+1}$ such that $\lim r_{i}=r>0$, and $\theta_{i}$ is not in any of the $S_{j}$ up to the $i$-th.

It remains to show that $r(\theta)=r$, as a priori only the inequality " $\leq$ " is known. Buff and Chéritat demonstrate it in their context. The idea is, roughly speaking, 
in showing that the boundary of $\Delta\left(\theta_{i}\right)$ is well approximated by a periodic cycle of a high period. The perturbation $\theta_{i} \mapsto \theta_{i+1}$ is then chosen sufficiently small so that the cycle does not move much.

As a final remark, let us point out:

Remark 5.1. Combining the methods of BC1 with our argument as outlined here one may strengthen the Main Theorem by showing that there exists a noncomputable Siegel Julia set for which the boundary of the Siegel disk is smooth.

Further progress. It is a natural question to ask whether the construction of non-computable Julia sets carried out in this paper can be replaced with a different, perhaps, simpler approach. Jointly with I. Binder, we have demonstrated the following in BBY1]:

Theorem 5.1 ([BBY1]). Let $R$ be a rational mapping of the Riemann sphere with no rotation domains (either Siegel disks or Herman rings). Then its Julia set is computable by a TM $M^{\phi}$ with an oracle for the coefficients of $R$.

Moreover, it is shown in the same paper that the Julia set of a quadratic polynomial $J_{c}$ with a periodic Siegel disk with conformal radius $r$ is computable by a TM with an oracle for $c$ if and only if $r$ itself is computable by some such machine. In retrospect, therefore, our approach finds the only available class of examples.

The size of the set of parameter values $\theta \in \mathbb{R} / \mathbb{Z}$ for which $J\left(P_{\theta}\right)$ is noncomputable is rather meagre. One can show combining the results of [BBY1] with, for example, those of Petersen and Zakeri $\mathrm{PZ}$ that this set has Lebesgue measure zero; and Theorem 5.1 implies that its complement contains a dense $G_{\delta}$ subset of $\mathbb{R} / \mathbb{Z}$. It is natural to ask if, for example, its Hausdorff dimension is positive, and the answer to this question is not known to us. It is also interesting to ask if any values of $\theta$ in this set are computable reals (as there are only countably many computable reals, and our procedure clearly produces an uncountable set of $\theta$ 's, most of them cannot be computable). We again do not know the answer to this.

On the practical side of things, to our knowledge, one has not been able to produce informative pictures of quadratic Julia sets with Cremer orbits, although by Theorem 5.1 this is theoretically possible. One potential explanation is that the computational complexity of these sets (the amount of time it takes to decide whether to color a pixel of size $2^{-n}$ as a function of $n$ ) is very high. This is indeed so for the naïve algorithms. In BBY2] jointly with I. Binder we have constructed quadratic Julia sets whose computational complexity is arbitrarily high, but again all with Siegel disks.

A natural first step towards studying the complexity of Cremer Julia sets is to look at parabolics, but the first author has recently demonstrated in Brv2 that having a parabolic orbit does not qualitatively change the complexity of computing a Julia set. This opens an entertaining possibility that some Cremer Julia sets have attainable computational complexity and could be practically drawn by a clever algorithm.

\section{ACKNOWLEDGEMENT}

We are grateful to John Milnor for his encouragement, and for many helpful suggestions on improving the exposition. We wish to thank Arnaud Chéritat for helpful comments on an earlier draft of this paper. The first author would like to thank Stephen Cook for many discussions on computability of real sets. 


\section{REFERENCES}

[BCSS] L. Blum, F. Cucker, M. Shub, S. Smale, Complexity and Real Computation, Springer, New York, 1998. MR:1479636 (99a:68070)

[ABC] A. Avila, X. Buff, A. Chéritat. Siegel disks with smooth boundaries. Acta Math. 193 (2004), no. 1, 1-30. MR 2155030

[BBY1] I. Binder, M. Braverman, M. Yampolsky. Filled Julia sets with empty interior are computable, e-print math.DS/0410580 at Arxiv.org.

[BBY2] I. Binder, M. Braverman, M. Yampolsky. On computational complexity of Siegel Julia sets, Commun. Math. Physics, to appear.

[BB] E. Bishop, D.S. Bridges, Constructive Analysis. Springer-Verlag, Berlin, 1985. MR0804042 (87d:03172)

[Brv1] M. Braverman, Computational Complexity of Euclidean Sets: Hyperbolic Julia Sets are Poly-Time Computable, Proc. CCA 2004., Electr. Notes Theor. Comput. Sci. 120: 17-30 (2005).

[Brv2] M. Braverman, Parabolic Julia sets are polynomial time computable. e-print math.DS/0505036 at Arxiv.org.

[Bru] A.D. Brjuno. Analytic form of differential equations, Trans. Mosc. Math. Soc. 25(1971), 119-262. MR0377192 (51:13365)

[BC1] X. Buff, A. Chéritat, Quadratic Siegel disks with smooth boundaries. Preprint Univ. Paul Sabatier, Toulouse, III, Num. 242.

[BC2] X. Buff, A. Chéritat, The Yoccoz function continuously estimates the size of Siegel disks, Annals of Math., to appear.

[Do] A. Douady. Does a Julia set depend continuously on the polynomial? In Complex dynamical systems: The mathematics behind the Mandelbrot set and Julia sets. ed. R.L. Devaney, Proc. of Symposia in Applied Math., Vol. 49, Amer. Math. Soc., 1994, pp. 91-138. MR 1315535

[DH1] A. Douady, J.H. Hubbard. Etude dynamique des polynômes complexes, I-II. Pub. Math. d'Orsay, 1984. MR0762431 (87f:58072a) MR0812271 (87f:58072b)

[DH2] A. Douady, J.H. Hubbard. On the dynamics of polynomial-like mappings. Ann. Sci. École Norm. Sup., 18(1985), 287-343. MR0816367 (87f:58083)

[Grz] A. Grzegorczyk, Computable functionals, Fund. Math. 42, pp. 168-202, 1955. MR0086756 (19:238b)

[Ko1] K. Ko, Complexity Theory of Real Functions, Birkhäuser, Boston, 1991. MR1137517 (93i:03057)

[Ko2] K. Ko, Polynomial-time computability in analysis, in "Handbook of Recursive Mathematics", Volume 2 (1998), Recursive Algebra, Analysis and Combinatorics, Yu. L. Ershov et al. (Editors), pp. 1271-1317. MR1673610(2000e:03169)

[Lac] D. Lacombe, Extension de la notion de fonction récursive aux fonctions d'une ou plusieurs variables réelles. I, C. R. Acad. Sci. Paris 240 (1955), 2478-2480; Extension de la notion de fonction récursive aux fonctions d'une ou plusieurs variables réelles. II, III, C. R. Acad. Sci. Paris 241 (1955), 13-14, 151-153. MR0072079(17:225d), MR0072080(17:225e)

[Maz] S. Mazur, Computable analysis, Rosprawy Matematyczne, Warsaw, vol. 33 (1963). MR0153553(27:3517)

[MMY] S. Marmi, P. Moussa, J.-C. Yoccoz, The Brjuno functions and their regularity properties, Commun. Math. Phys. 186(1997), 265-293. MR1462766 (98e:58137)

[Mat] Y. Matiyasevich, Hilbert's Tenth Problem, The MIT Press, Cambridge, London, 1993. MR.1244324 (94m:03002b)

[McM1] C. McMullen. Complex dynamics and renormalization. Annals of Math. Studies, v.135, Princeton Univ. Press, 1994. MR1312365 (96b:58097)

[Mil] J. Milnor. Dynamics in one complex variable. Introductory lectures. Friedr. Vieweg \& Sohn, Braunschweig, 1999. MR1721240 (2002i:37057)

[PZ] C. Petersen, S. Zakeri, On the Julia set of a typical quadratic polynomial with a Siegel disk. Ann. of Math. (2) 159(2004), no. 1, 1-52. MR2051390(2005c:37085)

[Pom] C. Pommerenke, Boundary behaviour of conformal maps, Springer-Verlag, 1992. MR 1217706 (95b:30008)

[Ret] R. Retinger. A fast algorithm for Julia sets of hyperbolic rational functions, Proc. of CCA 2004, Electr. Notes Theor. Comput. Sci. 120: 145-157 (2005). 
[RW] R. Rettinger, K. Weihrauch, The Computational Complexity of Some Julia Sets, in STOC'03, June 9-11, 2003, San Diego, California, USA. MR2121048 (2005j:68060)

[RZ] S. Rohde, M. Zinsmeister, Variation of the conformal radius, Journ. Anal. Math., 92(2004), pp. 105-115. MR2072743 (2005g:30008)

[Sie] C. Siegel, Iteration of analytic functions. Ann. of Math. (2) 43, (1942), 607-612. MR0007044 (4:76c)

[Sip] M. Sipser, Introduction to the Theory of Computation, PWS Publishing Company, 1997.

[Tur] A. M. Turing, On Computable Numbers, With an Application to the Entscheidungsproblem. In Proceedings, London Mathematical Society, 1936, pp. 230-265.

[Wei] K. Weihrauch, Computable Analysis, Springer, Berlin, 2000. MR.1795407 (2002b:03129)

[Yoc] J.-C. Yoccoz, Théorème de Siegel, nombres de Bruno et polynômes quadratiques, Petits diviseurs en dimension 1, S.M.F., Astérisque, 231(1995), 3-88. MR.1367353 (96m:58214)

Department of Computer Science, University of Toronto, Toronto, ON M5S 3G4, CANADA

Department of Mathematics, University of Toronto, Toronto, ON M5S 2E4, Canada 